\title{
From 3D dualities to hadron physics
}

\author{
Naoto Kan, ${ }^{1,2}$ Ryuichiro Kitano, ${ }^{1,2}$ Shimon Yankielowicz, ${ }^{3}$ and Ryo Yokokura ${ }^{1,4}$ \\ ${ }^{1}$ KEK Theory Center, Tsukuba 305-0801, Japan \\ ${ }^{2}$ Graduate University for Advanced Studies (Sokendai), Tsukuba 305-0801, Japan \\ ${ }^{3}$ The Raymond and Beverly Sackler School of Physics and Astronomy, Tel Aviv University, \\ Ramat Aviv 69978, Israel \\ ${ }^{4}$ Department of Physics \& Research and Education Center for Natural Sciences, Keio University, \\ Hiyoshi 4-1-1, Yokohama, Kanagawa 223-8521, Japan
}

(Received 22 June 2020; accepted 8 December 2020; published 30 December 2020)

\begin{abstract}
When one spacetime dimension is compactified on $S^{1}$, QCD exhibits a chiral phase transition at some critical radius. When we further turn on a background $\theta$ term which depends on the $S^{1}$ compactified coordinate, a topological ordered phase appears at low energy via the winding of $\theta$. We discuss what kind of theories can describe the physics near the critical point by requiring the matching of topological field theories in the infrared. As one of the possibilities, we propose a scenario where the $\rho$ and $\omega$ mesons form a $U\left(N_{f}\right)$ gauge theory near the critical point. In the phase where chiral symmetry is restored, they become the dual gauge boson of the gluon related by the level-rank duality between the three-dimensional gauge theories $S U(N)_{N_{f}}$ and $U\left(N_{f}\right)_{-N}$.
\end{abstract}

DOI: 10.1103/PhysRevD.102.125034

\section{INTRODUCTION}

The low-energy limit of QCD is described by pions whose properties and interactions have information about the global symmetry of QCD. The lowest-dimensional interaction terms can be determined once we know the coset space of which the pions are the coordinate. Also, the inconsistency in gauging a part of the global symmetry, i.e., the 't Hooft anomaly is encoded in the Wess-Zumino (WZ) term in the low-energy effective Lagrangian $[1,2]$.

The long-distance behavior is also important near the critical point of a phase transition. If the phase transition is smooth enough, one can consider an effective theory of an order parameter which obtains a vacuum expectation value (VEV) in the broken phase. Global structures of QCD such as anomalies should also be kept in the effective theory for consistency of the theory. Moreover, the 't Hooft anomaly results in matching conditions that constrain the realization of the vacuum structure and the infrared degrees of freedom [3-5]. In the case of finite-temperature QCD above the QCD scale, such an anomaly matching is usually trivially satisfied. The finite-temperature system can be regarded as an $S^{1}$ compactified QCD, and therefore the effective theory is a three-dimensional theory that has no chiral anomaly.

Published by the American Physical Society under the terms of the Creative Commons Attribution 4.0 International license. Further distribution of this work must maintain attribution to the author(s) and the published article's title, journal citation, and DOI. Funded by SCOAP ${ }^{3}$.
The study of phase transitions in three-dimensional gauge theory has a long history [6-16], with some important recent developments [17-31]. Even though there is no chiral anomaly, there are topological orders in the lowenergy effective theories. For example, $S U(N)$ gauge theory can have a Chern-Simons (CS) term with an integer level $k$. The CS term dominates the infrared physics, and it reduces to the CS theory [denoted as $S U(N)_{k}$ ] which has a gap, but the Wilson lines have nontrivial values depending on their topology. This nontrivial topological behavior should be matched when we discuss the effective description near the phase transition. Based on this discussion, the dualities between CS theories coupled to fermions and those coupled to bosons have been proposed and checked. The precise forms of the dualities are listed in Ref. [18]. Based on these dualities, the phase diagrams of threedimensional QCD $\left(\mathrm{QCD}_{3}\right)$ have been discussed [24]. In particular, it has been conjectured that the $S U(N)_{k}$ theory with $N_{f}(>2|k|)$ fermions undergoes the symmetry breaking $U\left(N_{f}\right) \rightarrow U\left(N_{f} / 2+k\right) \times U\left(N_{f} / 2-k\right)$ when the fermion masses are smaller than some critical value. There is a phase transition between the symmetry broken and unbroken phases as the fermion masses are varied. If the transition is of second order, near the critical point there is a dual description of the theory by the $U\left(N_{f} / 2+k\right)_{-N}$ or $U\left(N_{f} / 2-k\right)_{N}$ theory coupled to $N_{f}$ scalar fields whose Higgs phenomenon describes the phase transition.

In the unbroken phase in $\mathrm{QCD}_{3}$ the $\mathrm{CS}$ theory describes the low-energy physics. The fermionic theory flows to the $S U(N)_{ \pm N_{f} / 2+k}$ theory, while it is the $U\left(N_{f} / 2 \pm k\right)_{-N}$ 
theory in the bosonic theory. These two theories are related by the level-rank duality [32-35] and are known to give the same physics [36]. It is rather nontrivial that the matching of the low-energy limit is realized in this way.

In this paper, motivated by the symmetry breaking and its dual description in $\mathrm{QCD}_{3}$, we discuss the low-energy limits of $S^{1}$ compactified QCD in four spacetime dimensions $[24,37-70]$ with the hope that the three-dimensional duality may give us some hints about the four-dimensional physics. Indeed, for Abelian gauge theories it has been pointed out that the three-dimensional duality is lifted up to the $S$ duality in the four-dimensional theory [71]. We consider a background $\theta$ term which depends on the coordinate of the $S^{1}$ direction. In particular, the function $\theta$ can have windings along the $S^{1}$ direction [72,73], which determines the CS level in the three-dimensional effective theory. As the most interesting example, one can take the background with the winding number $N_{f}$, which is the number of quark flavors in four dimensions. For a small radius, one finds that the low-energy theory is $S U(N)_{N_{f}}$ where the vacuum is gapped. At a large radius, the theory is better described by the chiral Lagrangian for pions with the WZ terms. Since the low-energy limits are different, there must be a phase transition at some critical radius. The background $\theta$ induces a winding of the $\eta^{\prime}$ meson, which gives rise to the nontrivial WZ term in the threedimensional theory. The two limits of the theory can be interpolated by the Higgs mechanism of the $U\left(N_{f}\right)_{-N}$ theory coupled to $2 N_{f}$ flavors of scalar fields. Here, again, in the unbroken phase the theory is related by the level-rank duality. If this picture is correct, the natural candidates for these dual $U\left(N_{f}\right)$ gauge bosons are the $\rho$ and $\omega$ mesons (in the spirit of Ref. [74]), which we know phenomenologically to be successfully described in terms of gauge fields [75-81]. See also Refs. [82-84] for the interpretation of the Seiberg duality as the gauge theory of vector mesons, and Refs. [85,86] for the relation between the Seiberg duality and the phase structure of $\mathrm{QCD}_{3}$. The "gauge bosons" are massive anyway by the CS term even in the unbroken phase in the three-dimensional effective theory. Therefore, they are not quite effective degrees of freedom, but their existence is important for having the nontrivial topological order required from the matching of the lowenergy physics.

We discuss the similarities between $\mathrm{QCD}_{3}$ for small $k$ and $\mathrm{QCD}_{4}$, and propose an exotic possibility that the $\rho$ and $\omega$ mesons continuously becoming a dual gauge boson of gluons related by the level-rank duality. This conjectured picture is at least consistent when the winding of $\theta$ is less than $N_{f}$, especially when it is zero, in which case the system can be regarded as finite-temperature QCD.

The paper is organized as follows. In Sec. II we discuss the pattern of flavor symmetry breaking in $\mathrm{QCD}_{3}$ with $2 N_{f}$ fermions and its breaking to the chiral symmetry group associated with $\mathrm{QCD}_{4}$. We identify the order parameter that leaves the correct Nambu-Goldstone bosons massless to match the one of $\mathrm{QCD}_{4}$. The corresponding effective lowenergy theory is written down with an emphasis on the CS terms and the related WZ terms, also in the presence of external gauge fields. In particular, we identify the term associated with baryon number. These terms are related to the flavor anomalies in $\mathrm{QCD}_{4}$. They were also recently discussed by Komargodski in Ref. [28]. In Sec. III we put $\mathrm{QCD}_{4}$ on $M^{3} \times S^{1}$ including a $\theta$ angle which winds along $S^{1}$ and background gauge fields which depend on the $S^{1}$ coordinate. We explore the theory for small radius $\left(\Lambda_{4} R \ll 1\right)$ and large radius $\left(\Lambda_{4} R \gg 1\right)$ and argue for the existence of a phase transition at some critical $R_{*}$. In $\mathrm{Sec}$. IV we speculate/conjecture on the possible behavior of the hadronic vector mesons $(\rho, \omega, \ldots)$ near the critical point and the nature of the theory at the critical point. In particular, we put forward a scenario in which the hadronic vector mesons become gauge bosons and give rise to a $U\left(N_{f}\right)$ gauge theory at the critical point. In Sec. V we present holographic QCD-like models represented by quiver diagrams which capture this conjectured scenario of vector mesons as gauge bosons. In Sec. VI we comment briefly on the implications of our study for finite-temperature QCD and possible scenarios for the nature of the phase transition at $T_{*}=1 / R_{*}$. Lattice simulations may decide between these various scenarios. Section VII is devoted to discussion. There are three Appendices. In Appendix A we write down the Lagrangian associated with the quiver diagram in Sec. V. Appendix B addresses the issue of the integration over $S^{1}$ when a winding $\theta$ term is present. In Appendix $\mathrm{C}$ we discuss the issue of the baryon number and (winding) configuration of $\eta^{\prime}$ by considering the WZ term and the associated anomaly of the chiral $U(1)_{A}$ current, $U(1)_{\mathrm{EM}}$ electromagnetic current, and $U(1)$ baryon number. In particular, we can consider the situation that $\eta^{\prime}$ winding happens on a finite sheet and is localized on $S^{1}$. The sheet configuration is just the Hall droplet studied in Ref. [28]. Following Ref. [28], we can identify the baryonic configuration that resides on the boundary of the finite region. It is "amusing" to note that one can also identify a nonlocal configuration which corresponds to the quark with its Wilson line going into the bulk. In this sense the quark appears as a "soliton" in the hadronic effective theory.

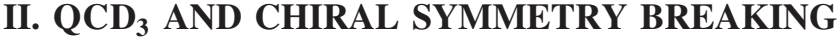

Based on the studies of the three-dimensional dualities, it has been conjectured that the low-energy theory of threedimensional $S U(N)_{0}$ QCD with $2 N_{f}$ fermions is described by a nonlinear sigma model with the target space

$$
\frac{U\left(2 N_{f}\right)}{U\left(N_{f}\right) \times U\left(N_{f}\right)}
$$


for small enough fermion masses $[24,87]$. There is an upper bound on $N_{f}$, although the precise location is unknown [88]. It is noted that an appropriate WZ term should be added to the Lagrangian. The same low-energy theory is obtained by a linear sigma model with $U\left(N_{f}\right)_{N}$ or $U\left(N_{f}\right)_{-N}$ gauge theory coupled to $2 N_{f}$ scalar fields. Beyond the critical value of the fermion mass, both the fermionic and bosonic theories flow to topological field theories related by the level-rank duality, $S U(N)_{ \pm N_{f}} \leftrightarrow U\left(N_{f}\right)_{\mp N}$.

We would like to discuss the relation between these symmetry-breaking phenomena and the chiral symmetry breaking in $\mathrm{QCD}_{4}$. In $\mathrm{QCD}_{4}$ with $N_{f}$ Dirac fermions (corresponding to $2 N_{f}$ fermions in $\mathrm{QCD}_{3}$ ), the low-energy theory is a nonlinear sigma model with

$$
\frac{S U\left(N_{f}\right)_{L} \times S U\left(N_{f}\right)_{R}}{S U\left(N_{f}\right)_{L+R}} .
$$

This coset space is a subspace of Eq. (1). One can reduce the space (1) to Eq. (2) by adding explicit breaking terms in the Lagrangian.

In this section we investigate and discuss the physics of $\mathrm{QCD}_{3}$ deformed by an explicit breaking term to exhibit a similar symmetry-breaking pattern as $\mathrm{QCD}_{4}$. Throughout this section we stay in three-dimensional spacetime, and analyze the phase structure near the critical quark mass. Later, in Secs. III and IV, we will compare and relate the phase structure of the deformed $\mathrm{QCD}_{3}$ revealed in this section to the critical phase transition in $\mathrm{QCD}_{4}$ compactified on a circle. ${ }^{1}$

In what follows, we explicitly break the $U\left(2 N_{f}\right)$ symmetry of $\mathrm{QCD}_{3}$ by hand, $U\left(2 N_{f}\right) \rightarrow U\left(N_{f}\right)_{L} \times U\left(N_{f}\right)_{R}$, and then discuss the spontaneous breaking $U\left(N_{f}\right)_{L} \times U\left(N_{f}\right)_{R} \rightarrow$ $U\left(N_{f}\right)_{L+R}$. We denote the $N_{f}+N_{f}$ flavors of $\mathrm{QCD}_{3}$ by $\psi$ and $\tilde{\psi}$, and introduce the explicit breaking term by coupling a massive adjoint scalar field $a_{3}$ to $\mathrm{QCD}_{3}$,

$$
\mathcal{L}_{a_{3}}=-\bar{\psi} a_{3} \psi+\overline{\tilde{\psi}} a_{3} \tilde{\psi}
$$

This interaction breaks the $U\left(2 N_{f}\right)$ symmetry to $U\left(N_{f}\right) \times U\left(N_{f}\right)$. In $\mathrm{QCD}_{4}$, the role of $a_{3}$ is played by the extra component of the gauge boson.

If we do not introduce the explicit breaking term in Eq. (3), the symmetry-breaking pattern is described in Eq. (1). This breaking pattern suggests that the order parameter is

$$
\langle\bar{\psi} \psi-\overline{\tilde{\psi}} \tilde{\psi}\rangle
$$

\footnotetext{
${ }^{1}$ Later in this section, we will comment on the relation of a deformed version of $\mathrm{QCD}_{3}$ to $\mathrm{QCD}_{4}$ compactified on a circle. This will allow us to borrow and use some techniques which are familiar in $\mathrm{QCD}_{4}$ for the investigation of the deformed $\mathrm{QCD}_{3}$.
}

and/or

$$
\langle\bar{\psi} \tilde{\psi}\rangle .
$$

Both VEVs reproduce the symmetry pattern $U\left(2 N_{f}\right) \rightarrow$ $U\left(N_{f}\right) \times U\left(N_{f}\right)$ as they are actually equivalent by $U\left(2 N_{f}\right)$ flavor rotation.

Once the $U\left(2 N_{f}\right)$ breaking terms in Eq. (3) are introduced, as long as $a_{3}$ is heavy enough, at least one of the VEVs in Eqs. (4) and (5) should remain nonvanishing. They are no longer equivalent, and which VEV remains nonvanishing is a dynamical issue. Indeed, the symmetry-breaking pattern is different. If $\langle\bar{\psi} \tilde{\psi}\rangle$ is nonvanishing, the $U\left(N_{f}\right) \times U\left(N_{f}\right)$ symmetry is spontaneously broken down to $U\left(N_{f}\right)$, while there is no symmetry breaking if it vanishes, as $\langle\bar{\psi} \psi-\tilde{\tilde{\psi}} \tilde{\psi}\rangle$ is invariant under $U\left(N_{f}\right) \times U\left(N_{f}\right)$.

We argue that $\langle\bar{\psi} \psi-\overline{\tilde{\psi}} \tilde{\psi}\rangle=0$ should be chosen at the massless point, and thus for small fermion masses the symmetry-breaking pattern is $U\left(N_{f}\right) \times U\left(N_{f}\right) \rightarrow U\left(N_{f}\right)$. One can see the symmetry-breaking pattern by analyzing the effective Lagrangian after integrating out the massive scalar field $a_{3}$. At the classical level, the effective Lagrangian can be evaluated as

$$
\begin{aligned}
\mathcal{L}_{\text {eff }, a_{3}}= & -\frac{1}{8 M^{2}}\left((\bar{\psi} \psi)^{2}+(\overline{\tilde{\psi}} \tilde{\psi})^{2}-2|\bar{\psi} \tilde{\psi}|^{2}\right) \\
& -\frac{1}{4 M^{2} N}(\bar{\psi} \psi-\overline{\tilde{\psi}} \tilde{\psi})^{2}+\cdots,
\end{aligned}
$$

where $M$ is the mass of $a_{3}$, and the ellipsis “..." denotes terms given by $\left(\bar{\psi} \gamma^{\mu} \psi\right)\left(\bar{\psi} \gamma_{\mu} \psi\right), \quad\left(\overline{\tilde{\psi}} \gamma^{\mu} \tilde{\psi}\right)\left(\overline{\tilde{\psi}} \gamma_{\mu} \tilde{\psi}\right)$, and $\left(\bar{\psi} \gamma^{\mu} \tilde{\psi}\right)\left(\overline{\tilde{\psi}} \gamma_{\mu} \psi\right)$. The effective potential in Eq. (6) implies that the potential energy increases if the VEV in Eq. (4) is nonzero, and decreases if the VEV in Eq. (5) is nonzero under the assumption that $\left\langle(\bar{\psi} \psi)^{2}\right\rangle,\left\langle(\overline{\tilde{\psi}} \tilde{\psi})^{2}\right\rangle$, and $\left\langle(\bar{\psi} \tilde{\psi})^{2}\right\rangle$ can be approximately written as $\langle\bar{\psi} \psi\rangle^{2},\langle\overline{\tilde{\psi}} \tilde{\psi}\rangle^{2}$, and $\langle(\bar{\psi} \tilde{\psi})\rangle^{2}$, respectively.

The discussion in the last paragraph lacks rigor. To make a more precise argument, we use the fact that $\mathrm{QCD}_{3}$ deformed by Eq. (3) can be obtained as the lowest Kaluza-Klein (KK) mode of $\mathrm{QCD}_{4}$ compactified on a circle in a metastable vacuum in a weakly coupled regime, namely, at a small radius. In this weakly coupled regime, we can control the validity of our approach. More precisely, the three-dimensional model with the explicit breaking term in Eq. (3) can be obtained as the low-energy effective theory of the $S^{1}$ compactified $\mathrm{QCD}_{4}$ with $N_{f}$ Dirac fermions, $\Psi$, as we will see in the next section. For the current discussion, one can take the $\theta$ term in $\mathrm{QCD}_{4}$ to be absent in order to match the CS level, $k=0$, in $\mathrm{QCD}_{3}$. We will discuss the case with a nonzero $\theta$ term in the next section. As is shown in Fig. 1 of Sec. III, the model with massless fermions can be realized at a metastable vacuum 

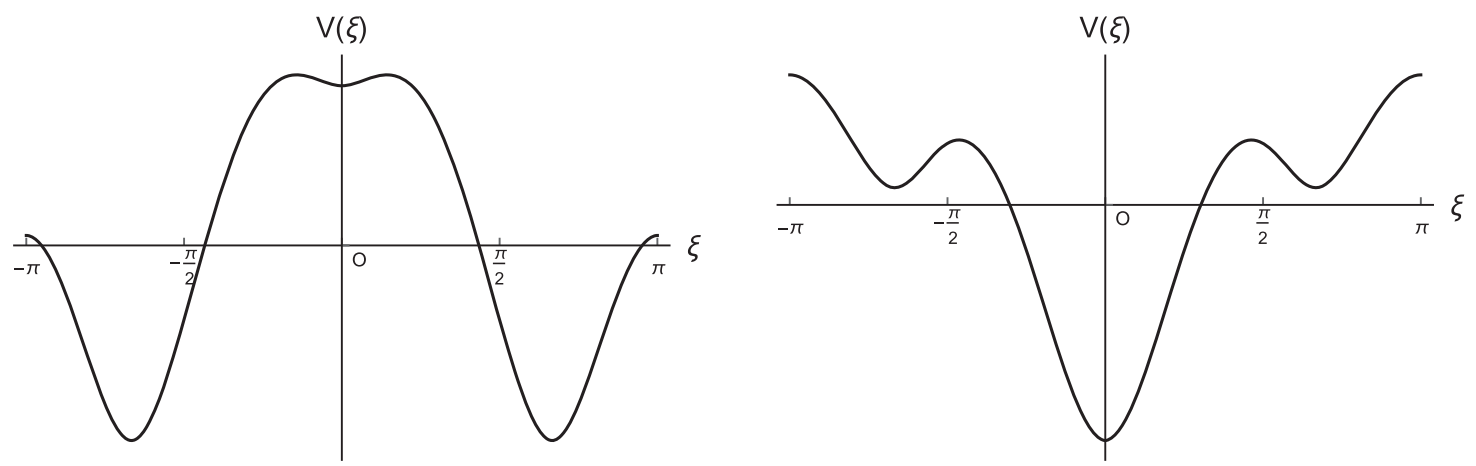

FIG. 1. Shapes of the potential at $\nu=0$ (left) and $\nu=\pi$ (right).

where the Wilson loop is trivial, $\left\langle e^{i \int a_{3}}\right\rangle=\mathbf{1}$, when $N_{f}<N$. The periodic boundary condition is taken for $\Psi{ }^{2}$ In this language, $\langle\bar{\psi} \psi-\overline{\tilde{\psi}} \tilde{\psi}\rangle$ and $\langle\bar{\psi} \tilde{\psi}\rangle$ correspond to $\left\langle\bar{\Psi} \gamma^{3} \Psi\right\rangle$ and $\langle\bar{\Psi} \Psi\rangle$, respectively, where $x_{3}$ is the $S^{1}$ direction.

Let us consider the Euclidean theory on a torus (which has no $\theta$ term), for which the path integral measure is nonnegative. We will further take the periodic boundary condition for $\Psi$ along the $x_{4}$ direction in addition to the $x_{3}$ direction. If there is a metastable vacuum with $\left\langle\bar{\Psi} \gamma_{3} \Psi\right\rangle \neq 0$, there should also be a metastable vacuum with a nonvanishing fermion density, $\left\langle\bar{\Psi} \gamma_{4} \Psi\right\rangle \neq 0$. This follows from the fact that this Euclidean theory with radii $\left(R_{3}, R_{4}\right)$ along the $\left(x_{3}, x_{4}\right)$ directions is equivalent to the one with radii $\left(R_{4}, R_{3}\right)$ along $\left(x_{3}, x_{4}\right)$, since we take the same periodic boundary conditions along these directions.

Let us assume that $\left\langle\bar{\Psi} \gamma_{4} \Psi\right\rangle \neq 0$ in the vacuum, and show that it leads to a contradiction. The following discussion is essentially identical to the Vafa-Witten theorem [89,90]. The nonzero $\left\langle\bar{\Psi} \gamma_{4} \Psi\right\rangle$ corresponds to nonzero charge density $\left\langle\bar{\Psi} \gamma_{0} \Psi\right\rangle$ in the Minkowski spacetime. We will deform the Minkowskian action by adding the term $\mu \bar{\Psi} \gamma_{0} \Psi$. Here, $\mu$ is a real chemical potential, and we assume that $|\mu|$ is sufficiently small so that higherorder perturbations of the free energy in $\mu$ can be neglected, and the metastability of the vacua is not violated. Note that the VEV $\left\langle\bar{\Psi} \gamma_{0} \Psi\right\rangle$ is nonzero in the limit $\mu \rightarrow 0$ by assumption. In the presence of a small but nonzero $\mu$, the free energy becomes $\mu\left\langle\bar{\Psi} \gamma_{0} \Psi\right\rangle+E_{0}$ to lowest order in $\mu$, where $E_{0}$ is the free energy at $\mu=0$. In general, $E_{0}$ depends on the VEV of the Wilson loop which labels metastable and stable vacua. Since $\left\langle\bar{\Psi} \gamma_{0} \Psi\right\rangle$ can be positive or negative, there is a vacuum where $\mu\left\langle\bar{\Psi} \gamma_{0} \Psi\right\rangle$ is negative, hence lowering the energy.

Now we show that the presence of the nonzero VEV $\left\langle\bar{\Psi} \gamma_{0} \Psi\right\rangle$ is impossible by using the expression in the Euclidean path integral. The term $\bar{\Psi} \gamma_{0} \Psi$ in the Minkowski

\footnotetext{
${ }^{2}$ Note that the fermions become massive in the true vacuum.
}

spacetime corresponds to $\bar{\Psi} \gamma_{4} \Psi$ in the Euclidean space. The above deformation by the chemical potential in the Minkowski spacetime corresponds to the deformation by adding $\mu \bar{\Psi} \gamma_{4} \Psi$ in the Euclidean action. In the Euclidean $\mathrm{QCD}_{4}$, however, since the path integral measure is non-negative [89] while adding a chemical potential gives a phase, the free energy cannot decrease with $\mu$ and has (for small $\mu$ ) its minimal value at vanishing chemical potential, i.e., $\mu=0$. Therefore, having a nonzero VEV $\left\langle\bar{\Psi} \gamma_{4} \Psi\right\rangle$ contradicts the non-negativity of the path integral. This means that $\left\langle\bar{\Psi} \gamma_{4} \Psi\right\rangle=0$ for any radius of the $x_{4}$ direction, implying that also $\left\langle\bar{\Psi} \gamma_{3} \Psi\right\rangle=0$, in contradiction to our assumption $\left\langle\bar{\Psi} \gamma_{3} \Psi\right\rangle \neq 0$.

We emphasize that the conclusion holds when we restrict the path integral to the background with $\left\langle e^{i \int a_{3}}\right\rangle=\mathbf{1}$ which leads to a metastable vacuum. Our argument relies only on the non-negative measure of the path integral which holds in any Euclidean background of the gauge field. This enables us to analyze the vacuum structure of $\mathrm{QCD}_{3}$ and conclude that $\mathrm{QCD}_{3}$ with massless $2 N_{f}$ flavors with no CS term $(k=0)$ (thus having a non-negative measure) and explicit breaking term is realized as the low-energy effective theory of $\mathrm{QCD}_{4}$ with $N_{f}$ flavors, with $\theta=0$ compactified on $S^{1}$ at a metastable vacuum.

Next, we consider a limit to match the three-dimensional theory. By the KK expansion of $\left\langle\bar{\Psi} \gamma^{3} \Psi\right\rangle$, the integral of the $\mathrm{VEV}$ along the $S^{1}$ direction is given by

$$
\int_{0}^{2 \pi R} d x_{3}\left\langle\bar{\Psi} \gamma^{3} \Psi\right\rangle=\langle\bar{\psi} \psi-\overline{\tilde{\psi}} \tilde{\psi}\rangle+\sum_{n \neq 0}\left\langle\bar{\psi}_{n} \psi_{n}-\overline{\tilde{\psi}}_{n} \tilde{\psi}_{n}\right\rangle,
$$

where $\psi_{n}$ and $\tilde{\psi}_{n}$ denote the $n$th KK modes. The VEV $\langle\bar{\psi} \psi-\overline{\tilde{\psi}} \tilde{\psi}\rangle$ in the three-dimensional theory corresponds to one of the lowest $(n=0) \mathrm{KK}$ modes. As we will see in Sec. III, one can have a finite energy gap between the mass scale of the first KK mode, $1 / R$, and the dynamical scale of $\mathrm{QCD}_{3}$ when the theory is weakly coupled at the compactification scale. Therefore, there exists a limit where higher KK modes are decoupled, and only the zero modes 
participate in the three-dimensional dynamics. In this limit, we have $\int_{0}^{2 \pi R} d x_{3}\left\langle\bar{\Psi} \gamma^{3} \Psi\right\rangle=\langle\bar{\psi} \psi-\overline{\tilde{\psi}} \tilde{\psi}\rangle$. By using $\left\langle\bar{\Psi} \gamma^{3} \Psi\right\rangle=0$, we obtain

$$
\langle\bar{\psi} \psi-\overline{\tilde{\psi}} \tilde{\psi}\rangle=0 .
$$

Therefore, in the three-dimensional limit, $\langle\bar{\psi} \tilde{\psi}\rangle \neq 0$ should be chosen at the massless point and the same symmetrybreaking pattern is expected for small fermion masses.

For $\langle\bar{\psi} \tilde{\psi}\rangle \neq 0$ in the presence of the explicit breaking terms in Eq. (3), the $U\left(N_{f}\right) \times U\left(N_{f}\right)$ chiral symmetry is broken to $U\left(N_{f}\right)$, leaving a part of the Nambu-Goldstone modes massless and matching $\mathrm{QCD}_{4}$ up to an anomalous axial $U(1)_{A}{ }^{3}$. Therefore, it is possible that the chiral symmetry breaking in $\mathrm{QCD}_{4}$ has something to do with the phase transition in $\mathrm{QCD}_{3}$ where there is a dual description by the Higgs mechanism of $U\left(N_{f}\right)_{ \pm N}$ gauge theory. In $\mathrm{QCD}_{4}$, it is well known that the physics of the vector mesons $\rho$ and $\omega$ is nicely described by the colorflavor locked phase of $U\left(N_{f}\right)$ gauge theory. The structure of the dual bosonic theory in three dimensions is indeed of this type, as is revealed in the following discussion based on the low-energy effective theory.

Let us look at the low-energy effective theory. We first discuss the theory without the explicit breaking terms in Eq. (3). In this case, the nonzero $\operatorname{VEV~}\langle\bar{\psi} \tilde{\psi}\rangle$ breaks $U\left(2 N_{f}\right)$ to $U\left(N_{f}\right) \times U\left(N_{f}\right)$. In the dual $U\left(N_{f}\right)_{ \pm N}$ bosonic theory, this symmetry breaking corresponds to a colorflavor locked phase: the $U\left(N_{f}\right)$ gauge symmetry is completely Higgsed, and the $U\left(2 N_{f}\right)$ flavor symmetry is broken, while a part of $U\left(2 N_{f}\right)$ flavor rotation together with a $U\left(N_{f}\right)$ gauge rotation is unbroken. The unbroken flavor symmetry is $U\left(N_{f}\right) \times U\left(N_{f}\right)$. The pions in this symmetry breaking have the WZ term as discussed in Ref. [24]. The WZ term can be obtained from the CS term in the bosonic $U\left(N_{f}\right)_{ \pm N}$ theory. The uneaten $2 N_{f}^{2}$ NambuGoldstone fields in Eq. (1) are introduced as a $2 N_{f} \times 2 N_{f}$ matrix:

$\xi=\exp \left[\frac{i}{\sqrt{2}} \pi^{a}\left(\begin{array}{cc}0 & T^{a} \\ T^{a} & 0\end{array}\right)+\frac{i}{\sqrt{2}} \tilde{\pi}^{a}\left(\begin{array}{cc}0 & i T^{a} \\ -i T^{a} & 0\end{array}\right)\right]$,

where $T^{a}$ are the generators of the $U\left(N_{f}\right)$ group. The Higgsed $U\left(N_{f}\right)$ gauge field $b_{\mu}$ couples to $\xi$ as

\footnotetext{
${ }^{3}$ In $\mathrm{QCD}_{4}$, the axial $U(1)_{A}$ symmetry is also broken by the axial anomaly. The corresponding Nambu-Goldstone mode, the $\eta^{\prime}$ meson, becomes massive and is decoupled from the lowenergy effective theory. In three dimensions, we can explicitly break the $U(1)_{A}$ symmetry by adding $|\log \operatorname{det} \overline{\tilde{\psi}} \psi|^{2}$, which corresponds to $|\log \operatorname{det} \xi|^{2}$ in the low-energy effective theory. Since the $\eta^{\prime}$ meson becomes massless in the large- $N$ limit, our discussion is valid at large $N$.
}

$$
\mathcal{L}_{\mathrm{NLSM}}=\frac{f^{2}}{4}\left|\partial_{\mu} \xi+i \xi\left(\begin{array}{cc}
b_{\mu} & 0 \\
0 & 0
\end{array}\right)\right|^{2}
$$

In the low-energy limit the gauge field $b_{\mu}$ can be integrated out, since this gauge field is massive by the Higgs mechanism. The equation of motion for $b_{\mu}$ gives

$$
b_{i j}=\left(\xi^{-1} d \xi\right)_{i j}
$$

where $i, j=1, \ldots, N_{f}$ run the first half of the $2 N_{f}$ indices. Substituting this into the CS term,

$$
S_{\mathrm{CS}}= \pm \frac{N}{4 \pi} \int_{M^{3}} \operatorname{Tr}\left(b d b+\frac{2}{3} b^{3}\right)
$$

one obtains the WZ terms.

We now introduce the explicit breaking term in Eq. (3). Half of the pions obtain masses when we include the interaction to break the $U\left(2 N_{f}\right)$ symmetry to the chiral symmetry. For example, one can introduce a spurion field,

$$
X=\left(\begin{array}{ll}
0 & \mathbf{1} \\
\mathbf{1} & 0
\end{array}\right)
$$

and write down a $U\left(2 N_{f}\right)$-breaking term,

$$
\operatorname{Tr}\left(\xi^{-1} X \xi X\right)
$$

This term gives a mass to $\tilde{\pi}$ while leaving $\pi$ massless.

In the low-energy limit where $\tilde{\pi}^{a}$ are decoupled, one can set $\tilde{\pi}^{a}=0$ and the WZ term among pions vanishes. However, one can trace the existence of the WZ term by turning on external gauge fields. Let us introduce the background gauge fields for the unbroken $U\left(N_{f}\right)$ global symmetry $A^{\mu}$, as the one which couples to the $U\left(N_{f}\right)$ vector current, $\bar{\psi} \gamma^{\mu} T^{a} \psi+\overline{\tilde{\psi}} \gamma^{\mu} T^{a} \tilde{\psi}$. The $U(1)$ part is the baryon number. The equation of motion for $b$ now gives $b=A+\cdots$, and thus we have

$$
S_{\mathrm{WZ}}= \pm \frac{N}{4 \pi} \int_{M^{3}} \operatorname{Tr}\left(A d A+\frac{2}{3} A^{3}\right)+\cdots
$$

In particular, we have a term

$$
\pm \frac{1}{4 \pi} \int_{M^{3}} B \operatorname{Tr}(d A)
$$

where $B$ is the baryon number normalized such that the quarks have the charge $1 / N$. The above term gives the baryon number $(B=1)$ for a monopole that has the unit magnetic charge of the $U(1)$ subgroup of $U\left(N_{f}\right)$. For example, the monopole made from the (11) component of $b$ has baryon number $B=1$. 


\section{III. $\mathrm{QCD}_{4}$ ON A CIRCLE}

We discuss the four-dimensional QCD compactified on $S^{1}$ and look for a relation to the phase transition in $\mathrm{QCD}_{3}$. We start with the action of $S U(N)$ gauge theory coupled with $N_{f}$ massless Dirac fermions, $\Psi_{i},\left(i=1, \ldots, N_{f}\right)$, with the assumption that $N>N_{f}$ on $M^{3} \times S^{1}$,

$$
\begin{aligned}
S= & \int_{M^{3} \times S^{1}} d^{4} x\left[-\frac{1}{2 g_{4}^{2}} \operatorname{Tr}\left(f_{M N} f^{M N}\right)\right. \\
& +\frac{\theta\left(x_{3}\right)}{32 \pi^{2}} \epsilon_{M N P Q} \operatorname{Tr}\left(f^{M N} f^{P Q}\right)+i \bar{\Psi}_{i} \gamma^{M}\left(\partial_{M}-i a_{M}\right) \Psi_{i} \\
& \left.-\partial_{M} \alpha_{L}^{i}\left(x_{3}\right) \bar{\Psi}_{i} \gamma^{M} P_{L} \Psi_{i}-\partial_{M} \alpha_{R}^{i}\left(x_{3}\right) \bar{\Psi}_{i} \gamma^{M} P_{R} \Psi_{i}\right] .
\end{aligned}
$$

The periodic boundary conditions are imposed on gauge fields. The Lorentz indices $M, N, P, Q$ run from 0 to 3 , where $x_{3}$ is the $S^{1}$ direction. $P_{L, R}$ are projection operators of chirality, $P_{L, R}=\left(1 \mp \gamma_{5}\right) / 2$. We introduced the $x_{3}$-dependent background fields $\theta\left(x_{3}\right)$ and $\alpha_{L, R}^{i}\left(x_{3}\right)$. The boundary condition of the quarks are

$$
\Psi_{i}\left(x_{3}+2 \pi R\right)=e^{i \nu} \Psi_{i}\left(x_{3}\right),
$$

where $0 \leq \nu<2 \pi$.

The $S^{1}$ compactification requires that $\theta$ and $\alpha$ are also valued on $S^{1}$, which allows

$$
\int_{S^{1}} d \theta=2 \pi k, \quad \int_{S^{1}} d \alpha_{L, R}^{i}=2 \pi m_{L, R}^{i},
$$

where $k$ and $m_{L, R}^{i}$ are integers. The integral on $S^{1}$ should be properly defined (as in Ref. [72]) so that the partition function does not depend on the coordinate system on $S^{1}$. (See Appendix B for the definition.) There is a redundancy due to the anomalous chiral symmetry, $\Psi_{L(R) i} \rightarrow$ $e^{i \beta_{L(R)}^{i} \Psi_{L(R) i},}$

$$
\begin{aligned}
\theta\left(x_{3}\right) & \rightarrow \theta\left(x_{3}\right)-\sum_{i}\left(\beta_{R}^{i}\left(x_{3}\right)-\beta_{L}^{i}\left(x_{3}\right)\right), \\
\alpha_{L, R}^{i}\left(x_{3}\right) & \rightarrow \alpha_{L, R}^{i}\left(x_{3}\right)+\beta_{L, R}^{i}\left(x_{3}\right),
\end{aligned}
$$

where

$$
\int_{S^{1}} d \beta_{L, R}^{i}=2 p \pi,
$$

where $p$ is an integer to maintain the boundary conditions.

In the following discussion, we are particularly interested in the theory with

$$
k=N_{f}, \quad m_{L, R}^{i}=0
$$

since the vacuum structure looks the same as the threedimensional case discussed in the previous section.
By the chiral rotations, this theory is equivalent to, for example,

$$
k=0, \quad m_{R}^{i}=1, \quad m_{L}^{i}=0 .
$$

The physics should depend on the combination:

$$
\begin{aligned}
\bar{\theta}\left(x_{3}\right) & =\theta\left(x_{3}\right)+\sum_{i}\left(\alpha_{R}^{i}\left(x_{3}\right)-\alpha_{L}^{i}\left(x_{3}\right)\right), \\
\bar{k} & =k+\sum_{i}\left(m_{R}^{i}-m_{L}^{i}\right) .
\end{aligned}
$$

We discuss the phase structure of the theory as a function of the radius $R$. The dynamical scale $\Lambda_{4}$ is defined as

$$
\Lambda_{4}^{b}=\Lambda^{b} e^{-8 \pi^{2} / g_{4}^{2}(\Lambda)}, \quad b=\frac{11}{3} N-\frac{2}{3} N_{f},
$$

where $\Lambda$ is an arbitrarily high scale. For $\Lambda_{4} R \gg 1$, the lowenergy dynamics is described by hadrons on an $S^{1}$ compactified background. In the other limit, $\Lambda_{4} R \ll 1$, the low-energy description is a three-dimensional gauge theory on $M^{3}$ via the KK decomposition. The gauge coupling constant in the three-dimensional effective theory is given by

$$
\frac{1}{g_{3}^{2}}=\frac{2 \pi R}{g_{4}^{2}(1 / R)}
$$

and the dynamical scale in the three-dimensional theory is defined as

$\Lambda_{3}=\frac{g_{3}^{2} N}{8 \pi}=\frac{g_{4}^{2}(1 / R) N}{16 \pi^{2} R}=\frac{1}{R}\left(-\frac{2 b}{N} \log \left(\Lambda_{4} R\right)\right)^{-1}$.

One can see that for a small enough $\Lambda_{4} R$, there is an energy gap between the dynamical scale $\Lambda_{3}$ and the mass of the first KK mode, $1 / R$.

\section{A. Small radius, $\Lambda_{\mathbf{4}} R \ll 1$}

Let us use the basis with $k=0$. The KK expansion of the fermions can be done as

$$
\Psi\left(x, x_{3}\right)=\sum_{n=-\infty}^{\infty}\left(\begin{array}{c}
\psi_{n}(x) \phi_{n}\left(x_{3}\right) \\
\sigma^{3} \tilde{\psi}_{n}(x) \tilde{\phi}_{n}\left(x_{3}\right)
\end{array}\right)
$$

with

$$
\phi_{n}\left(x_{3}\right)=\frac{1}{\sqrt{2 \pi R}} \exp \left[i \alpha_{L}\left(x_{3}\right)+i\left(\frac{n}{R}+\frac{m_{L}}{R}+\frac{\nu}{2 \pi R}\right) x_{3}\right],
$$

and 
$\tilde{\phi}_{n}\left(x_{3}\right)=\frac{1}{\sqrt{2 \pi R}} \exp \left[i \alpha_{R}\left(x_{3}\right)+i\left(\frac{n}{R}+\frac{m_{R}}{R}+\frac{\nu}{2 \pi R}\right) x_{3}\right]$.

The three-dimensional effective action is given by

$$
\begin{aligned}
S_{\mathrm{eff}}= & \int d^{3} x\left[-\frac{1}{2 g_{3}^{2}} \operatorname{Tr}\left(f_{\mu \nu} f^{\mu \nu}\right)\right. \\
& +\sum_{n} i \bar{\psi}_{n} \gamma^{\mu}\left(\partial_{\mu}-i a_{\mu}\right) \psi_{n}+\sum_{n} i \overline{\tilde{\psi}}_{n} \gamma^{\mu}\left(\partial_{\mu}-i a_{\mu}\right) \tilde{\psi}_{n} \\
& -\sum_{n}\left(-\frac{m_{L}}{R}-\frac{n}{R}-\frac{\nu}{2 \pi R}\right) \bar{\psi}_{n} \psi_{n} \\
& -\sum_{n}\left(\frac{m_{R}}{R}+\frac{n}{R}+\frac{\nu}{2 \pi R}\right) \overline{\tilde{\psi}}_{n} \tilde{\psi}_{n} \\
& \left.+\frac{1}{g_{3}^{2}} \operatorname{Tr}\left(D_{\mu} a_{3} D^{\mu} a_{3}\right)-\left(\bar{\psi} a_{3} \psi-\overline{\tilde{\psi}} a_{3} \tilde{\psi}\right)-V\left(a_{3}\right)\right] .
\end{aligned}
$$

Here $V\left(a_{3}\right)$ is the effective potential for the gauge field along the $S^{1}$ direction, which we will determine in Eq. (34). We have dropped the massive KK modes of gauge fields. At this stage, one can see that the effects of $m_{L}$ and $m_{R}$ are to shift the KK spectrum of the fermions by $1 / R$, and thus they can be absorbed by the redefinition of $n$. However, in three dimensions the signs of the fermion masses are important, and thus one cannot simply ignore $m_{L, R}$.

We will show that the action in Eq. (31) describes a gapped phase. In particular, all of the fermions obtain masses by the nonzero VEV of $a_{3}$, while the VEV preserves the $S U(N)$ gauge symmetry. We will indicate the role of $a_{3}$ after the compactification. In particular, we determine the surviving gauge symmetry $H \subseteq S U(N)$ after the compactification. ${ }^{4}$

Let us consider a Wilson line around $S^{1}$,

$$
W=\mathcal{P} \exp \left(i \int_{0}^{2 \pi R} d x_{3} a_{3}\right)
$$

which can generally be diagonalized as $\operatorname{diag}\left(e^{i \xi_{1}}, e^{i \xi_{2}}, \ldots\right.$, $\left.e^{i \xi_{N}}\right)$, with $\sum_{i}^{N} \xi_{i}=0(\bmod 2 \pi)$.

The VEV of $a_{3}$ contributes to the mass of the KK modes of gauge bosons as follows:

$$
M_{n, i j}=\frac{1}{R}\left(n-\frac{\xi_{i}-\xi_{j}}{2 \pi}\right)
$$

If $\xi_{i} \neq \xi_{j}(\bmod 2 \pi)$, the KK mass spectrum breaks the $S U(N)$ gauge symmetry ${ }^{5}[53,92]$.

\footnotetext{
${ }^{4}$ The center symmetry of $S U(N)$ is explicitly broken by the fermions. For more on the center symmetry, see, e.g., Ref. [91].

${ }^{5}$ In other words, if the Wilson line $W$ and the generators of $S U(N)$ commute, the gauge symmetry is unbroken, $H=S U(N)$.
}

The phases $\left\{\xi_{i}\right\}$ are determined dynamically by the potential $V\left(a_{3}\right)$, which is generated at the one-loop level and depends on the boundary condition $\nu$. The one-loop effective potential as a function of the $\xi$ 's is given by

$$
\begin{aligned}
V\left(a_{3}\right)= & \frac{1}{8 \pi^{5} R^{3}}\left[-2 \sum_{i, j=1}^{N} \sum_{n=1}^{\infty} \frac{\cos n\left(\xi_{i}-\xi_{j}\right)}{n^{4}}\right. \\
& \left.+2 \sum_{i=1}^{N} \sum_{n=1}^{\infty} \frac{\cos n\left(-\xi_{i}+\nu+2 \pi m_{L}\right)}{n^{4}}+(L \rightarrow R)\right] .
\end{aligned}
$$

The potential is minimized when

$$
a_{3}=\frac{1}{2 \pi R} \operatorname{diag}(\xi, \xi, \ldots, \xi,-(N-1) \xi) .
$$

Inserting Eq. (35) into Eq. (34), we obtain

$$
\begin{aligned}
& V\left(a_{3}\right)=\frac{1}{8 \pi^{5} R^{3}}\left[-4(N-1) \sum_{n=1}^{\infty} \frac{\cos n N \xi}{n^{4}}\right. \\
& +2 N_{f}(N-1) \sum_{n=1}^{\infty}\left(\frac{\cos n\left(-\xi+\nu+2 \pi m_{L}\right)}{n^{4}}+(L \rightarrow R)\right) \\
& \left.\quad+2 N_{f} \sum_{n=1}^{\infty}\left(\frac{\cos n\left((N-1) \xi+\nu+2 \pi m_{L}\right)}{n^{4}}+(L \rightarrow R)\right)\right] .
\end{aligned}
$$

The shape of the potential of $\xi$ at $\nu=0(\nu=\pi)$ is shown in the left (right) panel of Fig. 1 for $N=3$ and $N_{f}=2$. In general, for $N_{f}<N$ there are $N$ minima at $\xi= \pm 2 p \pi / N$, $(p=0, \ldots,[N / 2])$. (For even $N, \xi=\pi$ and $\xi=-\pi$ are equivalent.) At $\nu=0, \xi=0$ is a local minimum, and the true minimum is at $\xi= \pm(N-1) \pi / N$ for odd $N$ and $\xi=\pi$ for even $N$. For $\nu=\pi$, the $\xi=0$ point is the true vacuum.

The potential has a symmetry $\nu \rightarrow \nu+2 \pi n / N, n \in \mathbb{Z}$, together with $\xi \rightarrow \xi-2 \pi n / N$ which is the reflection of the fact that the action of $\mathbb{Z}_{N}$ elements in $U(1)_{B}$ is the same as that of the gauge group $S U(N)$ [93]. For even $N, \nu=0$ and $\xi=\pi$ is equivalent to $\nu=\pi$ and $\xi=0$. For odd $N$, they are not equivalent. The $\nu=\pi$ point is equivalent to $\nu=\pi / N$ by an appropriate shift of $\xi$. There is a first-order transition in between $\nu=0$ and $\nu=\pi / N$.

In all of the $N$ minima, the $S U(N)$ gauge symmetry is unbroken as the Wilson loop along the $x_{3}$ direction is a phase times the unit matrix. The fermion masses for $\psi_{n}$ and $\tilde{\psi}_{n}$ are, respectively,

$$
\begin{aligned}
& m_{n}^{(\psi)}=-\frac{n}{R}-\frac{\nu}{2 \pi R}-\frac{m_{L}}{R}+\frac{\xi}{2 \pi R}, \\
& m_{n}^{(\tilde{\psi})}=\frac{n}{R}+\frac{\nu}{2 \pi R}+\frac{m_{R}}{R}-\frac{\xi}{2 \pi R} .
\end{aligned}
$$


By following the global minimum of the potential, in the entire region of $\nu$, the fermion masses are nonvanishing. Therefore, the low-energy limit of four-dimensional QCD on $M^{3} \times S^{1}$ is $S U(N)$ pure gauge theories on $M^{3}$ for a small radius. There is a mass gap, but the low-energy limit can be a topological field theory. For $m_{R}=1$ and $m_{L}=0$, the fermion masses for $\tilde{\psi}_{n}$ are shifted by $1 / R$. The shift changes the sign of $N_{f}$ fermion masses from negative to positive. Therefore, the low-energy theory obtains the CS level $N_{f}\left(N_{f} / 2\right.$ to integrate in the negative ones, and another $N_{f} / 2$ to integrate out the positive ones).

The CS level is consistent with the result in the basis of $k=N_{f}$. The $\theta$ term can be expressed as

$$
\begin{aligned}
& \frac{1}{8 \pi^{2}} \int_{M^{3} \times S^{1}} \theta \operatorname{Tr}(f f) \\
& \quad=\frac{1}{8 \pi^{2}} \int_{M^{3} \times S^{1}} \operatorname{Tr}\left(a d a+\frac{2}{3} a^{3}\right) d \theta, \quad \bmod 2 \pi .
\end{aligned}
$$

Again, it is important that the integral on $S^{1}$ is properly defined [72]. Since $d \theta$ is single valued, one can naively use the right-hand side of the integral over $S^{1}$, which reduces to the CS term with the level $k$ for the lowest KK mode of the gauge field.

In summary, the low-energy limit of $\mathrm{QCD}_{4}$ on $M^{3} \times S^{1}$ with Eq. (22) for a small radius, $\Lambda_{4} R \ll 1$, is the topological field theory, $S U(N)_{N_{f}}$, that has the dual description given by $U\left(N_{f}\right)_{-N}$.

\section{B. Large radius, $\Lambda_{4} R \gg 1$}

One can also analyze the low-energy limit of $\mathrm{QCD}_{4}$ on $M^{3} \times S^{1}$ for a large radius as we know that the low-energy theory is described by pions. We discuss the effect of $\bar{\theta}\left(x_{3}\right)$ in the low-energy effective theory. In order to see the $\bar{\theta}\left(x_{3}\right)$ dependence of the theory, one needs to introduce the $\eta^{\prime}$ meson in addition to the massless pions. Note that a similar topic where there is a phase transition as a function of the gradient of $\bar{\theta}$ was established in Ref. [25].

The effective theory is given in terms of the $N_{f} \times N_{f}$ unitary matrix

$$
U=e^{i \pi^{a} T^{a}+i \eta^{\prime}}
$$

We implicitly assume here that $\eta^{\prime}$ behaves as a NambuGoldstone boson, which is true in the large- $N$ limit. Hence, the results are formally correct to the leading order in the $1 / N$ expansion. The field $U$ transforms as

$$
U \rightarrow g_{L}^{-1} U g_{R}
$$

under the $g_{L} \in S U\left(N_{f}\right)_{L}$ and $g_{R} \in S U\left(N_{f}\right)_{R}$ chiral transformations. Under the axial $U(1)_{A}$, it transforms as $U \rightarrow e^{2 i \beta} U$. The effective action is given by

$$
\begin{aligned}
S_{\mathrm{eff}} & =\int_{M^{3} \times S^{1}} d^{4} x \\
& \times\left[f_{\pi}^{2} \operatorname{Tr}\left|\partial_{\mu} U\right|^{2}-\frac{m_{\eta^{\prime}}^{2} f_{\pi}^{2}}{N_{f}}\left|\log \left(e^{-i \bar{\theta}} \operatorname{det} U\right)\right|^{2}+\cdots\right] .
\end{aligned}
$$

Here we have added the mass term of $\eta^{\prime}$, which is generated at the order of $1 / N$. The effect of the boundary condition $\nu$ can be taken into account by introducing a background gauge field for the baryon number, $(\nu / 2 \pi R) \bar{\Psi} \gamma^{3} \Psi$. The effect of this term appears in the WZ terms. The parameter $\nu$ couples to the topological current, i.e., the Skyrmions.

In what follows we show that $\eta^{\prime}$ develops a winding number due to the winding of $\bar{\theta}$. Here we consider the case $\bar{k}=N_{f}$ as before, and discuss the case with smaller values of $\bar{k}$ later. In this case we obtain the same WZ term as in the three-dimensional $U\left(N_{f}\right)_{-N}$ theory by integrating out $\eta^{\prime}$. At the linearized order, the equation of motion for $\eta^{\prime}$ is

$$
\frac{\partial^{2}}{\partial x_{3}^{2}} \eta^{\prime}=m_{\eta^{\prime}}^{2}\left(\eta^{\prime}-\frac{\bar{\theta}}{N_{f}}\right) .
$$

Here we have used the parametrization of $U$ in Eq. (39) and neglected the derivatives along the $x_{0,1,2}$ directions, which are irrelevant for the discussion here. For the periodicity of $\bar{\theta}$, the potential for $\eta^{\prime}$ has $N_{f}$ domains where $\eta^{\prime}-\bar{\theta} / N_{f}$ is minimized at $2 n \pi / N_{f}$ (where $n$ is an integer). A transition between two other domains requires a treatment beyond this effective theory. If we require that the shape of the function $\bar{\theta}$ is not very rapid so that the effective theory can be used, $\eta^{\prime}$ should stay in one of the domains to minimize the energy, which means that $\eta^{\prime}$ develops a winding number under Eq. (22):

$$
\eta^{\prime}\left(x_{3}+2 \pi R\right)=\eta^{\prime}\left(x_{3}\right)+2 \pi .
$$

This is consistent with the $S^{1}$ compactification. Note that the non-zero winding number of $\eta^{\prime}$ does not imply the jump of domains. We are treating $\eta^{\prime}$ as a heavy field and we are working within the effective theory. Also, the argument of the winding of $\eta^{\prime}$ depends on the basis. We can eliminate $\bar{\theta}$ by the field redefinition of $\eta^{\prime}$. In this basis $\eta^{\prime}$ does not develop a winding number, while we obtain the same physics at low energy, which we will discuss in the next paragraph after introducing the WZ term in Eq. (44).

The three-dimensional low-energy effective theory is the nonlinear sigma model with the coset in Eq. (2), but there are effects from the $\eta^{\prime}$ winding. By turning on the external gauge field $A$, which couples to the vector current (as we discussed in the previous section), a part of the WZ term,

$$
S_{\mathrm{WZ}}=-\frac{N}{8 \pi^{2}} \int_{M^{3} \times S^{1}} \operatorname{Tr}\left(A d A+\frac{2}{3} A^{3}\right) d \eta^{\prime},
$$


reduces to Eq. (15) where the minus sign is chosen, i.e., it is the same as the one obtained from $U\left(N_{f}\right)_{-N}$ theory. It is interesting that the external magnetic field carries baryon number in this $\eta^{\prime}$ winding background. See Appendix C for the relation between the profile of $\eta^{\prime}$ and the baryons. The parameter set in Eq. (23) corresponds to the basis where $\eta^{\prime}-\bar{\theta} / N_{f}$ is redefined to be $\eta^{\prime}$. In that basis there is no winding of $\eta^{\prime}$, but the same WZ term appears by shifting $\eta^{\prime}$ in Eq. (44). One can confirm the consistency of the appearance of the WZ term by comparing the $\bar{\theta} \rightarrow A A$ amplitude to that in $\mathrm{QCD}_{4}$.

In summary, we find that the low-energy limit of $\mathrm{QCD}_{4}$ with Eq. (22) is a topological field theory, $S U(N)_{N_{f}}$, for small $\Lambda_{4} R$, and a nonlinear sigma model with the WZ term for a large radius, $\Lambda_{4} R \gg 1$. There must be a phase transition between these two extreme regions. It is interesting to find that the two limits are the same as the conjectured limit of the three-dimensional $S U(N)_{0}$ theory with $2 N_{f}$ fermions with large and small fermion masses. It is therefore possible to anticipate that the phase transition between a large and small radius is described by the critical point of $S U(N)_{0} \mathrm{QCD}_{3}$ with $2 N_{f}$ fermions with the explicit $U\left(2 N_{f}\right)$ symmetry-breaking terms. The phase transition can also be consistent with the description by the three-dimensional $U\left(N_{f}\right)_{-N}$ theory with $2 N_{f}$ scalar fields.

For $|\bar{k}|<N_{f}$ instead of Eq. (22), the $\eta^{\prime}$ winding should be accompanied by the winding of pions. In order to satisfy the boundary condition, $U\left(x_{3}+2 \pi R\right)=U\left(x_{3}\right)$, the winding $\eta^{\prime}\left(x_{3}+2 \pi R\right)=\eta^{\prime}\left(x_{3}\right)+2 \pi \bar{k} / N_{f}$ should be accompanied by

$$
e^{i \pi^{a} T^{a}\left(x_{3}+2 \pi R\right)}=e^{-2 \pi i \bar{k} / N_{f}} e^{i \pi^{a} T^{a}\left(x_{3}\right)},
$$

which can be realized as a configuration of $\pi^{a}$ as the phase factor is an element of $S U\left(N_{f}\right)$. For example, for $\bar{k}=1$, one of the diagonal components of $U$ acquires a winding of $2 \pi$, and the nontrivial WZ term appears only for that component of the external gauge fields. In general, for $|\bar{k}| \leq$ $N_{f}$ the same effective three-dimensional theory can be obtained by the theories of pions interacting with a Higgsed $U(|\bar{k}|)_{\mp N}$ gauge group. This part matches the conjectured dualities in three dimensions between $S U(N)_{\bar{k}-N_{f}}$ with $2 N_{f}$ fermions and $U(\bar{k})_{-N}$ with $2 N_{f}$ scalars.

\section{HADRONS NEAR THE CRITICAL POINT}

Here we speculate on the possible behavior of the vector mesons based on the discussion in the previous section. As we discussed, $\mathrm{QCD}_{4}$ in the background of Eq. (22) provides us with the same low-energy theories of $\mathrm{QCD}_{3}$ in both the broken and unbroken phases of the global symmetry once an explicit breaking term of the $U\left(2 N_{f}\right)$ symmetry is added. The phase transition in three dimensions has a dual picture by the $U\left(N_{f}\right)_{-N}$ gauge theory. Below, we will consider the possibility that the dual picture also describes the $\mathrm{QCD}_{4}$ near the phase transition.

Indeed, it is interesting that the extension of the chiral Lagrangian to a $U\left(N_{f}\right)$ gauge theory is known to be very successful at describing the phenomenology of the vector mesons $\rho$ and $\omega$ [75-77]. Therefore, the vector mesons are the natural candidates for the gauge bosons of the $U\left(N_{f}\right)$ dual theory. If such an interpretation is true, in the phase where chiral symmetry is restored, the $\rho$ and $\omega$ mesons are in the topological phase rather than the Higgs phase under the background in Eq. (22).

The possible behavior of the hadrons as functions of the radius is as follows. Starting from a large radius where the hadrons describe physics effectively, as the radius approaches the critical point, $R_{*} \sim 1 / \Lambda_{4}$, the lowest mode of hadrons start to form a $U\left(N_{f}\right)_{-N}$ gauge theory in the Higgs phase. The members are pions, $\rho, \omega$ and other scalar mesons. At the critical point, the chiral symmetry is restored and the $\rho$ and $\omega$ mesons get into the topological phase described by the $U\left(N_{f}\right)_{-N}$ theory, which is dual to $S U(N)_{N_{f}}$. As the radius decreases further, the picture of weakly interacting gluons and quarks makes sense at the energy scale between $\Lambda_{3}$ and $1 / R$. All of the fermions as well as KK modes of gluons decouple below $1 / R$. Although the description in terms of hadrons becomes ineffective in this energy region, the low-energy limit of the theory stays the same.

Since the $U\left(N_{f}\right)$ gauge group is spontaneously broken, there are vortex configurations in three dimensions made of $\rho$ and $\omega$, which carry magnetic and electric charges of $U(1)^{N_{f}}\left[\subset U\left(N_{f}\right)\right]$ [94]. The electric charge is a consequence of the CS term. In the color-flavor locked phase, the electric charge is identified as the baryon number. The vortex with unit magnetic charge has $B=1$. We will discuss how this vortex configuration extends to the $S^{1}$ direction in the next section.

\section{HOLOGRAPHIC MODEL}

The vector mesons as gauge bosons are nicely described by the holographic QCD where the gauge bosons are propagating into an extra dimension [79-81]. Based on the holographic model, we here try to find a dual model of $\mathrm{QCD}_{4}$ that reproduces the story in the previous section. For the duality to work, we need the same low-energy limits in both the broken and unbroken phases of the chiral symmetry. In particular, one needs to arrange the theory such that there is an unbroken gauge group, $U\left(N_{f}\right)_{-N}$, in the symmetric phase. The chiral symmetry breaking is described by the VEVs of scalar fields, which simultaneously make the gauge group Higgsed.

Of course, we are not aware if the phase transition is smooth enough that such an effective description exists. Here we assume that the phase transition is of second order, 

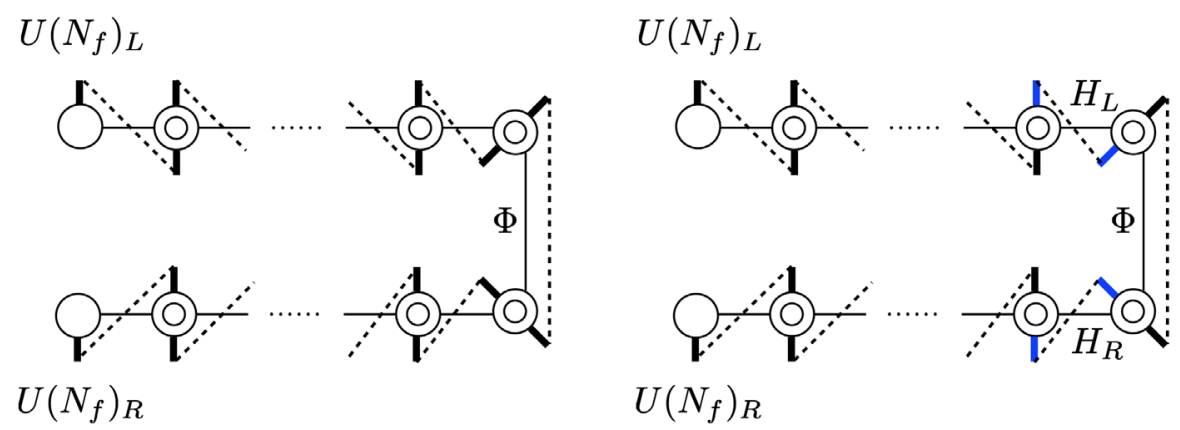

FIG. 2. Quiver diagram for holographic QCD (left) and the linearized model (right).

and look for a dual theory. ${ }^{6}$ In this sense, this is a construction of the Nambu-Jona-Lasino model or the Ginzburg-Landau model while taking into account the consistency with topology. For $\bar{k}=0$ it was a trivial task since the low-energy limit of the symmetric phase is trivially gapped. But for $\bar{k} \neq 0$ we need some gauge theory to survive to match the topological field theory. In this section we mainly consider the background in Eq. (22). We will comment on other backgrounds in the last part of this section.

Holographic QCD describes the vector mesons and pions as gauge fields propagating into the fifth dimension, $b_{L, R}$. The gauge group is $U\left(N_{f}\right)_{L} \times U\left(N_{f}\right)_{R}$, which is broken down to $U\left(N_{f}\right)_{L+R}$ somewhere in the extra dimension. The five-dimensional space has a boundary. The boundary conditions are taken to be

$$
\left.b_{L, R}^{\mu}\right|_{\text {boundary }}=A_{L, R}^{\mu}, \quad(\mu=0,1,2,3),
$$

where the right-hand side is external gauge fields which couple to chiral currents. The pions appear as the extradimensional component of $b_{L}^{4}-b_{R}^{4} \sim \partial^{4} \pi$. The boundary condition makes the gauge bosons massive, and the lightest modes are identified as the $\rho$ and $\omega$ mesons. The WZ terms can be reproduced by

$$
S_{\mathrm{CS}}=-\frac{N}{24 \pi^{2}} \int_{X_{5}}\left(\omega_{5}\left(b_{L}\right)-\omega_{5}\left(b_{R}\right)\right) .
$$

See Ref. [96] for details. The local chiral transformation shifts the external gauge fields and modifies the boundary conditions, which can be absorbed by the gauge transformation of the bulk gauge fields, and this in turn provides a boundary term from the gauge transformation of the CS term. This procedure results in the WZ terms on the

\footnotetext{
${ }^{6}$ In this paper, we consider the massless fermions with the periodic boundary condition along the $x_{3}$ direction, and the number of colors is assumed to be greater than the number of flavors, $N>N_{f}$. If we instead impose the antiperiodic boundary condition, the order of the transition would be changed (see, e.g., Ref. [95]).
}

boundary written in terms of the pions and the external gauge fields. They are necessary to match the 't Hooft anomaly in $\mathrm{QCD}_{4}$.

The deconstructed version of the model can be built as in the left panel of Fig. 2 [78]. The explicit form of the Lagrangian is given in Appendix A. The leftmost sites with open circles are the boundary. No gauge fields live there. At other sites with double circles, there are $U\left(N_{f}\right)$ gauge fields which are all Higgsed by eating the link fields. By the rightmost link, denoted $\Phi$, the $U\left(N_{f}\right)_{L} \times U\left(N_{f}\right)_{R}$ symmetry is broken down to $U\left(N_{f}\right)_{L+R}$. Since the number of links is larger by one than the number of double circles, there are $N_{f}^{2}$ Nambu-Goldstone bosons left uneaten. They are identified as the pions and $\eta^{\prime}$. The thick lines at each site represent $N$ chiral fermions. The dashed line between fermions represents the mass terms. They are all massive, but necessary to reproduce the CS term in the five-dimensional theory. Since the fermions are always massive even in the chiral-symmetric phase (as we will see later), we can think of fermions as auxiliary degrees of freedom. We also gauge the $\mathbb{Z}_{N}$ subgroup of $U(1)_{B}$ which transforms fermions. The gauging is necessary to match the global symmetry $U(1)_{B} / \mathbb{Z}_{N}$ in the original QCD. The gauging requires that one should sum up all of the sectors with boundary conditions of fermions twisted by elements of $\mathbb{Z}_{N}$ in the $x_{3}$ direction.

Now let us turn on the external gauge fields. The external gauge fields couple to fermions as

$$
A_{L}^{\mu}\left(\bar{q}_{L} \gamma_{\mu} q_{L}+\cdots\right)+(L \leftrightarrow R) .
$$

The fermions in the upper and lower wings couple to $A_{L}$ and $A_{R}$, respectively. The mass terms of fermions, except for the one with the link $\Phi$, do not break the global symmetry, $U\left(N_{f}\right)_{L} \times U\left(N_{f}\right)_{R}$. The combination of $A_{L}+$ $A_{R}$ couples to the conserved vector current. By integrating out the massive fermions, one obtains the correct WZ terms. At this stage, we have not included the mass term of $\eta^{\prime}$. One can introduce it by writing a mass term to break the $U(1)_{L-R}$ gauge symmetry for $\Phi$, such as

$$
|\log \operatorname{det} \Phi|^{2} .
$$


The axial $U(1)$ is now explicitly broken, and the $\eta^{\prime}$ obtains a mass. In the background of Eq. (17), we have the term

$$
\left|\log e^{-i \bar{\theta}} \operatorname{det}(\Phi)\right|^{2}
$$

This term causes the winding of the trace part of $\Phi$. The winding gives important effects through the WZ term, as we discussed before. In addition to the WZ terms among external fields and Nambu-Goldstone modes, we also have

$$
S_{\mathrm{WZ}}=-\frac{N}{8 \pi^{2}} \int_{M^{3} \times S^{1}} \operatorname{Tr}\left(b d b+\frac{2}{3} b^{3}\right) d(-i \log \operatorname{det} \Phi) / N_{f},
$$

where $b$ is a gauge field of the $U\left(N_{f}\right)_{L+R}$ part of the rightmost gauge sites. They are massive modes which correspond to the $\rho$ and $\omega$ mesons.

One can modify the model by introducing Higgs fields, $H_{L}$ and $H_{R}$, as in the right panel of Fig. 2. Two of the links are replaced by the Higgs fields. When the VEV of $\left\langle H_{L, R}\right\rangle$ (proportional to the unit matrix) is large, one can identify the sites on both sides of $H_{L, R}$, and we come back to the model in the left panel. The location of the links to be replaced with $H_{L, R}$ can be anywhere in the wings.

For small $\left\langle H_{L, R}\right\rangle$, the vector part of the gauge bosons in the rightmost sites, i.e., $\rho$ and $\omega$, becomes light, and for $\left\langle H_{L, R}\right\rangle=0$ the gauge bosons as well as the fermions (marked as blue lines) become "massless." They obtain masses when the $x_{3}$ direction is compactified on $S^{1}$. All of the link fields are eaten by the gauge fields, and thus massless pions disappear. Therefore, the chiral symmetry is now recovered in this phase. In this way, the model interpolates the chiral Lagrangian and the linear sigma model by changing the sizes of $\left\langle H_{L, R}\right\rangle$.

In the phase of $\left\langle H_{L, R}\right\rangle=0$, we have the term in Eq. (51) but now $b$ represents the massless gauge boson. For the background with $\bar{k}=N_{f}$, the winding of $\Phi$ gives the winding $\theta$ term for vector mesons.

Let us consider the vacuum of the theory after the compactification of the $x_{3}$ direction by the one-loop effective potential near the phase transition point, $\left\langle H_{L, R}\right\rangle=0$. In this region, the massless degrees of freedom are the $U\left(N_{f}\right)$ gauge field and $4 N$ chiral fermions. The scalar fields $H_{L, R}$ can be light, but the contribution of the scalars has the same shape as the fermions with the opposite sign, and thus for $N_{f}<N$ the effects can be ignored. Half of the $4 N$ massless fermions are charged under $U\left(N_{f}\right)$, while the rest are neutral. We first discuss the contribution to the effective potential of $b_{3}$ from the $2 N$ chiral fermions. We take an ansatz of the VEV of $b_{3}$ to be

$$
b_{3}=\frac{1}{2 \pi R} \operatorname{diag} \cdot\left(\tilde{\xi}_{1}, \tilde{\xi}_{2}, \ldots, \tilde{\xi}_{N_{f}}\right)
$$

where $\sum_{i=1}^{N_{f}} \tilde{\xi}_{i}=0(\bmod 2 \pi)$. The boundary condition of the fermions is

$$
q\left(x_{3}+2 \pi R\right)=e^{i \nu} q\left(x_{3}\right),
$$

where $0 \leq \nu<2 \pi$. The KK spectra of the gauge fields and the fermions are, respectively,

$$
\begin{aligned}
& M_{i j, n}^{2}=\frac{1}{R^{2}}\left(n-\frac{\tilde{\xi}_{i}-\tilde{\xi}_{j}}{2 \pi}\right)^{2}, \\
& m_{i, n}^{2}=\frac{1}{R^{2}}\left(n+\frac{\nu-\tilde{\xi}_{i}}{2 \pi}\right)^{2} .
\end{aligned}
$$

By using these, the one-loop effective potential is given by

$$
\begin{aligned}
V\left(b_{3}\right)= & \frac{1}{4 \pi^{5} R^{3}}\left(-\sum_{i, j=1}^{N_{f}} \sum_{n=1}^{\infty} \frac{\cos \left(n\left(\tilde{\xi}_{i}-\tilde{\xi}_{j}\right)\right)}{n^{4}}\right. \\
& \left.+2 N \sum_{i=1}^{N_{f}} \sum_{n=1}^{\infty} \frac{\cos \left(n\left(\nu-\tilde{\xi}_{i}\right)\right)}{n^{4}}\right) .
\end{aligned}
$$

The first term on the right-hand side is minimized when $\tilde{\xi}_{1}=\tilde{\xi}_{2}=\cdots=\tilde{\xi}_{N_{f}}=\tilde{\xi}$. Furthermore, the second term is minimized at $\tilde{\xi}=\nu-\pi$. Therefore, for $N_{f}<N$ the lowest minimum is at $\int_{S^{1}} b=(\pi-\nu) \cdot \mathbf{1}$, which gives the antiperiodic boundary conditions for the fermions. The $U\left(N_{f}\right)$ group is unbroken at the minimum, and the $2 N$ fermions become massive.

Next, we consider the one-loop effects of the gauged $\mathbb{Z}_{N}$ which is the subgroup of the $U(1)_{B}$ symmetry. The effective potential is given by

$$
V\left(\xi^{\prime}\right)=\frac{N}{2 \pi^{5} R^{3}} \sum_{n=1}^{\infty} \frac{\cos \left(n\left(\nu-\xi^{\prime}\right)\right)}{n^{4}},
$$

where $\xi^{\prime}=2 \pi m / N(m \in \mathbb{Z})$ is the VEV of the gauged $\mathbb{Z}_{N}$ field. The different VEV corresponds to the sector of different boundary conditions twisted by the $\mathbb{Z}_{N}$ elements. Since $\mathbb{Z}_{N}$ is gauged, we are summing up all of the values of $\xi^{\prime}$ in the path integral. The free energy is minimized at $m=N(\nu-\pi) /(2 \pi)$, which means the path integral is dominated by this sector. The antiperiodic boundary condition is chosen for even $N$, and the sector that is the closest to the antiperiodic one is chosen for odd $N$. Therefore, all of the fermions decouple and the effective three-dimensional theory is the $U\left(N_{f}\right)_{-N}$ CS theory where the CS level stems from the winding of $\Phi$. This theory is dual to $S U(N)_{N_{f}}$, which is the low-energy limit of $\mathrm{QCD}_{4}$ for a small $S^{1}$ radius.

It is important that the axial $U(1)$ remains broken in the phase of $\left\langle H_{L, R}\right\rangle=0$ where chiral symmetry $S U\left(N_{f}\right)_{L} \times$ $S U\left(N_{f}\right)_{R}$ is unbroken. The explicit breaking is important to 
obtain the correct CS level via the winding of $\Phi$. Therefore, in order for the scenario to work the axial $U(1)$ should be broken during the chiral phase transition. ${ }^{7}$

The baryon number as the topological charge in the $U\left(N_{f}\right)_{-N}$ theory can be seen in this model. The baryon number in this model is identified as the trace part of the external $U\left(N_{f}\right)_{L+R}$. The unbroken baryon number is rearranged to be a sum of all of the $U(1)$ parts of each site, and thus the fermions that are integrated out to obtain Eq. (51) are charged under the baryon number. Therefore, by turning on the background gauge field $B$, for the baryon number we obtain a term in the three-dimensional effective theory,

$$
S_{\text {baryon }}=-\frac{1}{4 \pi} \int_{M^{3}} B \operatorname{Tr}(d b),
$$

under the winding of $\Phi$. Here, we have integrated $\Phi$ in Eq. (51) in the presence of the winding number along the $x_{3}$ direction.

As in the three-dimensional effective picture, there are vortex configurations made of $\rho$ and $\omega$. Under the winding of $\Phi$, there is a winding $\theta$ term for the $U\left(N_{f}\right)$ gauge group from Eq. (51). In the presence of this $\theta$ term (which we call $\tilde{\theta}$ ), the Abrikosov-Nielsen-Olesen (ANO) vortex string $[99,100]$ which goes around $S^{1}$ cannot be connected since the magnetic flux obtains an electric charge as it goes around the $S^{1}$ direction by the Witten effect [101] (see Appendix $\mathrm{C}$ for details). In order to have a stable string loop that goes around $S^{1}$, one needs to have some nontrivial configuration which carries the electric charge, i.e., the baryon number.

In the background with a constant $d \tilde{\theta}$, one can look for a static field configuration that is $x_{3}$ independent. The field equations are then the same as in the CS case, and thus one finds the solution with a finite energy. The baryon number, $B=1$, is indeed carried by the string through the electric charge of this solution. For a general background, this configuration will be relaxed to a solution of the field equations with a finite energy. We call it the $B=1$ string.

Another possibility is to unwind $\Phi$, i.e., $\eta^{\prime}$ by forming a Hall droplet, as described in Ref. [28]. The droplet is a configuration of the $\eta^{\prime}$ field. It is a sheet with a boundary, and the value of $\eta^{\prime}$ changes by $\pm 2 \pi$ when we go across the sheet. The ANO $\rho-\omega$ string can be connected when it goes across the sheet as the Witten effect is canceled by the change of $\tilde{\theta}$. The net baryon number of this configuration is $B=0$, and thus we call it the $B=0$ string. This string penetrates the Hall droplet. The droplet cannot shrink to nothing, as there is no $B=0$ string in the background

\footnotetext{
${ }^{7}$ The breaking of the axial $U(1)$ symmetry implies that topological susceptibility does not vanish during the chiral phase transition (see, e.g., Refs. [97,98] for discussions following lattice simulations)
}

without the droplet. The $B=1$ string discussed above cannot smoothly deform into this string due to the different baryon number. Interestingly, it was proposed in Ref. [28] that the droplet has an excitation of the edge mode with $B=1$ and that object is identified as the baryon, such as $\Delta^{++} \sim u u u$. Therefore, the $B=1$ string can deform into a $B=0$ string together with the excitation of the edge mode of the droplet with $B=1$. Since there is no such stable string configuration in full $\mathrm{QCD}$, we expect that there are monopoles to cut the $B=0$ string. A pair of a monopole and an antimonopole can cut and eliminate the string. Now the $B=1$ string can decay into a baryon via the deformation into a $B=0$ string and a $B=1$ droplet, and then the $B=0$ string part is eliminated. This is a good candidate for the fate of the $B=1$ string.

So far, we have assumed the background in Eq. (22). The phase transition by the VEV of $H_{L, R}$ can be extended to the case of $0 \leq \bar{k}<N_{f}$. In that case, the unbroken gauge group is $U(\bar{k})_{-N} \times U\left(N_{f}-\bar{k}\right)_{0}$. In order for the theory to have the same low-energy limit as QCD, the $U\left(N_{f}-\bar{k}\right)_{0}$ factor should decouple. The $S U\left(N_{f}-\bar{k}\right)_{0}$ part exhibits a mass gap by the confinement. The $U(1)$ part also confines by instantons. As we discussed in the previous section, we need monopoles (in four dimensions) to cut the stable $\rho$ and $\omega$ strings. The monopole configurations in the (012) directions are instantons in three dimensions, and the path integral including such instantons causes the confinement of the $U(1)$ factor $[102,103]$. The presence of the monopole does not make the $U(\bar{k})_{-N}$ part confine as the gauge bosons have a mass term from the CS term [104]. Therefore, we obtain the correct low-energy limit, i.e., the $U(\bar{k})_{-N}$ theory.

There is another possibility that the $U\left(N_{f}-\bar{k}\right)$ part of the VEV is kept nonvanishing for $H_{L, R}$ while the chiral symmetry is restored by cutting the $U\left(N_{f}-\bar{k}\right)$ part of the link $\Phi$. The $U\left(N_{f}-\bar{k}\right)$ gauge group is kept in the Higgs phase, and the only $U(\bar{k})_{-N}$ part remains at low energy.

What happens for $\rho$ and $\omega$ mesons is qualitatively different in the above two cases. When the $S^{1}$ radius is large, they are vector mesons, which we are familiar with. As the radius approaches the critical point, they behave as the gauge bosons in the Higgs phase. In particular, the metastable vortex strings made of $\rho$ and $\omega$ appear. Beyond the critical radius the chiral symmetry is restored, and the $U(\bar{k})$ part of them goes into the topological phase, whereas the $U\left(N_{f}-\bar{k}\right)$ part either goes into the confining phase or remains in the Higgs phase. The rest of them stay in the Higgs phase.

The model described here can be viewed as the NambuJona-Lasino model for chiral symmetry breaking under the background of the imaginary chiral chemical potential (once we take the compactification direction to be the time direction in the Euclidean space.) The $\mathbb{Z}_{N}$ twisting boundary condition can be naturally identified as the VEV of the Polyakov loop. For a small radius (high temperature), 
$\left\langle H_{L, R}\right\rangle$ is vanishing, and thus the fermions choose a particular boundary condition by minimizing the free energy. This corresponds to the nonvanishing VEV of the Polyakov loop, and thus describes the deconfined phase. On the other hand, for a large radius where $\left\langle H_{L, R}\right\rangle$ is large, the fermions decouple, and all of the boundary conditions contribute equally to the path integral. This corresponds to a vanishing VEV for the Polyakov loop, and thus the quarks are confined.

\section{FINITE-TEMPERATURE QCD}

We discussed a somewhat exotic scenario for the chiral phase transition which happens at some critical radius $R_{*}$. Let us apply our results to finite-temperature QCD. Here we mainly consider our results for a general $\bar{k}$ in Eq. (24) rather than $\bar{k}=N_{f}$ in Eq. (22). In particular, we mainly discuss the $\bar{k}=0$ case, where the physical phase $\bar{\theta}$ is absent. For $\bar{k}=0$ with the antiperiodic boundary condition for quarks, $\nu=\pi$, one can think of this system as finite-temperature QCD by considering the Euclidean metric. At some critical temperature $T_{*}=1 / R_{*}$, the chiral phase transition happens.

In the model we discussed, there is a consistent scenario where $U(\bar{k})_{-N} \times U\left(N_{f}-\bar{k}\right)_{0}$ theory remains in the infrared and the $U\left(N_{f}-\bar{k}\right)_{0}$ factor confines due to instantons. For $\bar{k}=0$, the confining $U\left(N_{f}\right)_{0}$ gauge field is comprised of the $\rho$ and $\omega$ mesons.

There are other possibilities, as we discussed already. There is also a possibility that $U\left(N_{f}\right)$ gauge theory is not a good picture at all. For example, the gauged Nambu-JonaLasino model in Ref. [105] can give a $S U(N)_{\bar{k}}$ factor in the chiral-symmetric phase. The model simply adds a scalar field $X$ to QCD, which transforms as $\left(N_{f}, \bar{N}_{f}\right)$ under the $U\left(N_{f}\right)_{L} \times U\left(N_{f}\right)_{R}$ chiral symmetry and couples to quarks as $\bar{q} X q$. This model gives the correct nonlinear sigma model in the broken phase where the scalar field has a VEV, while it reduces to $S U(N)_{\bar{k}}$ theory in the symmetric phase. The $\rho$ and $\omega$ mesons do not appear as the field that describes the phase transition phenomena.

The question of which picture is the most appropriate near the chiral phase transition should be able to be tested by lattice simulations. By looking at the behavior of the two-point functions of the vector currents, one may check if the $\rho$ and $\omega$ mesons become "massless." However, this does not mean that the screening masses of the $\rho$ and $\omega$ mesons vanish. Although they have no mass term in the fourdimensional Lagrangian, they have thermal masses and also masses from instantons (monopoles in four dimensions) in the actual spectrum. We will leave the study of these effects as well as that of actual methods in lattice QCD to distinguish the scenarios to future work. For lattice simulations of their screening masses, see, e.g., Refs. [106-109].

\section{DISCUSSION}

Three-dimensional CS matter systems exhibit various nontrivial topological phases at low energy, and it has been conjectured that gauge theories with fermions and bosons describe the same physics near the critical point of the parameter spaces. Although this duality is tightly related to the peculiar anyon statistics in three spacetime dimensions, the symmetry-breaking phenomena and dualities conjectured in $\mathrm{QCD}_{3}$ with small CS levels look quite similar to our QCD vacuum in four dimensions.

The winding $\theta$ background on an $S^{1}$ compactified space can directly relate the three- and four-dimensional theories by comparing the low-energy limits. We found that in $\mathrm{QCD}_{4}$ the chiral phase transition should happen at a critical radius, and there can be a description of the phase transition as the Higgs mechanism of the $U\left(N_{f}\right)$ gauge theory where the gauge bosons are the vector mesons.

Under the winding $\theta$, the $\mathrm{WZ}$ terms in the chiral Lagrangian leave nontrivial WZ terms in the threedimensional effective theories, where the baryon number can be identified as the magnetic flux of QED. The origin of this unusual relation between baryons and monopoles can be understood as the 't Hooft anomaly in $\mathrm{QCD}_{4}$.

We left the discussion of how to test the possibility of the vector mesons becoming gauge bosons to future work. One of the natural frameworks to discuss this point is holographic QCD where the vector mesons are already gauge bosons in the Higgs phase. A specific holographic model such as the SakaiSugimoto model [79] may be used to study the dynamics of the phase transition in the winding $\theta$ background. Also, if the feature of gauge bosons becoming "massless" in the fourdimensional language remains in the trivial $\theta=0$ background, lattice QCD may be able to directly test the scenario.

Another nontrivial prediction of the model is the existence of the monopoles which cut the string made of $\rho$ and $\omega$ [110]. We are not sure what should be identified as these objects in the hadron spectrum. Due to the color-flavor locking, the monopoles really carry the magnetic charge of QED while they are confined by the string. It is certainly interesting to look for candidates for hadrons that are made of the monopole-string system.

\section{ACKNOWLEDGMENTS}

We would like to thank Ofer Aharony, Andreas Karch, Zohar Komargodski, Yutaka Sakamura, and Adi Armoni for discussions and useful comments. R. K. and S. Y. would like to thank the theory group at SLAC for hospitality during their stay. R. K. also thanks the theory group at UC Davis for warm hospitality during his stay. The work of R.K. is supported by JSPS KAKENHI Grants No. 15KK0176 and No. 19H00689, and MEXT KAKENHI Grant No. 18H05542. The work of S. Y. is supported in part by a Center of Excellence grant supported by the Israel Science Foundation (2289118) and the IsraelGermany Foundation (GIF). 


\section{APPENDIX A: LAGRANGIAN OF QUIVER DIAGRAM}

Here we show the Lagrangian that is described by the quiver diagram in the left panel of Fig. 2:

$$
\begin{aligned}
\mathcal{L}= & i \bar{q}^{(L) 0} \gamma^{M}\left(\partial_{M}-i A_{M}^{(L)}\right) P_{L} q^{(L) 0}+i \bar{q}^{(R) 0} \gamma^{M}\left(\partial_{M}-i A_{M}^{(R)}\right) P_{R} q^{(R) 0}+\sum_{i=1}^{n_{L}} i \bar{q}^{(L) i} \gamma^{M}\left(\partial_{M}-i b_{M}^{(L) i}\right) q^{(L) i} \\
& +\sum_{i=1}^{n_{R}} i \bar{q}^{(R) i} \gamma^{M}\left(\partial_{M}-i b_{M}^{(R) i}\right) q^{(R) i}-\sum_{i=1}^{n_{L}} \frac{1}{2 g_{i}^{(L) 2}} \operatorname{Tr}\left(f_{M N}^{(L) i} f^{(L) i M N}\right) \\
& -\sum_{i=1}^{n_{R}} \frac{1}{2 g_{i}^{(R) 2}} \operatorname{Tr}\left(f_{M N}^{(R) i} f^{(R) i M N}\right)+\operatorname{Tr}\left|\partial_{M} U_{01}^{(L)}-i A_{M}^{(L)} U_{01}^{(L)}+i U_{01}^{(L)} b_{M}^{(L) 1}\right|^{2} \\
& +\sum_{i=1}^{n_{L}-1} \operatorname{Tr}\left|\partial_{M} U_{i, i+1}^{(L)}-i b_{M}^{(L) i} U_{i, i+1}^{(L)}+i U_{i, i+1}^{(L)} b_{M}^{(L) i+1}\right|^{2}+\operatorname{Tr}\left|\partial_{M} U_{10}^{(R)}-i b_{M}^{(R)} U_{10}^{(R)}+i U_{10}^{(R)} A_{M}^{(R)}\right|^{2} \\
& +\sum_{i=1}^{n_{R}-1} \operatorname{Tr}\left|\partial_{M} U_{i+1, i}^{(R)}-i b_{M}^{(R) i+1} U_{i+1, i}^{(R)}+i U_{i+1, i}^{(R)} b_{M}^{(L) i}\right|^{2}+\operatorname{Tr}\left|\partial_{M} \Phi-i b_{M}^{(L) n_{L}} \Phi+i \Phi b_{M}^{(R) n_{R}}\right|^{2} \\
& -\sum_{i=0}^{n_{L}-1} m_{i, i+1}^{(L)} \bar{q}^{(L) i+1} U_{i, i+1}^{(L) \dagger} P_{L} q^{(L) i}-\sum_{i=0}^{n_{L}-1} m_{i+1, i}^{(R)} \bar{q}^{(R) i} U_{i+1, i}^{(R) \dagger} P_{L} q^{(R) i+1}+\text { H.c. }-m_{\Phi} \bar{q}^{(R) n_{R}} \Phi^{\dagger} P_{L} q^{(L) n_{L}}+\text { H.c. },
\end{aligned}
$$

where $n_{L}$ and $n_{R}$ are the number of double circle nodes in the upper and lower lines, respectively.

\section{APPENDIX B: WINDING $\theta$ TERM}

Here we review how to treat the $\theta$ term with a winding number [72]. When $\theta$ has a winding number along a compact direction, the $\theta$ term is not well defined on one patch.

Let us consider the integral

$$
\frac{1}{2 \pi} \int_{S^{1}} \theta d q
$$

with

$$
\int_{S^{1}} d \theta=2 \pi k, \quad \int_{S^{1}} d q=2 \pi n, \quad(k, n \in \mathbb{Z}) .
$$

We would like to define the integral up to $2 \pi m,(m \in \mathbb{Z})$ since the integral will be exponentiated in the path integral. In Ref. [72] a general prescription to define such an integral was discussed. By taking $t(0 \leq t<2 \pi)$ as the coordinate on $S^{1}$, the prescription gives

$$
\frac{1}{2 \pi} \int d t \theta(t) \dot{q}(t):=\frac{1}{2 \pi} \int_{0}^{2 \pi} d t \theta(t) \dot{q}(t)-k q(2 \pi)
$$

Similarly, the integration of $\dot{\theta}(t) q(t)$ can be defined by

$$
\frac{1}{2 \pi} \int d t \dot{\theta}(t) q(t):=\frac{1}{2 \pi} \int_{0}^{2 \pi} d t \dot{\theta}(t) q(t)-n \theta(2 \pi) .
$$

The definitions of the integral in Eqs. (B3) and (B4) have the following desired features. The integral is invariant modulo $2 \pi$ under the shifts $\theta \rightarrow \theta+2 \pi$ and $q \rightarrow q+2 \pi$. Also, the integral does not depend (modulo $2 \pi$ ) on the choice of the $t=0$ point on $S^{1}$.

These definitions are consistent with the integration by parts (modulo $2 \pi$ ), i.e.,

$$
\begin{aligned}
\frac{1}{2 \pi} \int d t \theta(t) \dot{q}(t) & =\frac{1}{2 \pi} \int_{0}^{2 \pi} d t \theta(t) \dot{q}(t)+k q(2 \pi) \\
& =-\frac{1}{2 \pi} \int_{0}^{2 \pi} d t \dot{\theta}(t) q(t)+n \theta(0) \\
& =-\frac{1}{2 \pi} \int d t \dot{\theta}(t) q(t), \quad \bmod 2 \pi .
\end{aligned}
$$

\section{APPENDIX C: BARYON NUMBER AND THE CONFIGURATION OF $\boldsymbol{\eta}^{\prime}$}

We discuss a configuration to give the baryon number $B \neq 0$ in QCD under a nontrivial background of $\eta^{\prime}$. We now take the spacetime as Minkowski space, $M^{4}$. The WZ term in QCD contains the following term:

$$
S_{\mathrm{WZ}}=-\frac{N}{8 \pi^{2}} \int \operatorname{Tr}\left(A d A+\frac{2}{3} A^{3}\right) d \eta^{\prime},
$$

where the $N_{f} \times N_{f}$ matrix $A$ is the external gauge field which couples to the $U\left(N_{f}\right)$ vector current, and $\eta^{\prime}$ is the $U(1)$ part of the Nambu-Goldstone mode, $U=e^{i \pi^{a} T^{a}+i \eta^{\prime}}$. The trace part of the gauge field, normalized as 
$B=\left(N / N_{f}\right) \operatorname{Tr} A$, is the source of the baryon number. The baryon charge density can be read off by differentiating with respect to $B$ as

$$
\rho_{B}=\frac{1}{4 \pi^{2}} \epsilon_{i j k} \operatorname{Tr}\left(\partial_{i} A_{j}\right) \partial_{k} \eta^{\prime}+\cdots .
$$

Let us consider a configuration with $\eta^{\prime}=0$ at $x_{3}=-\infty$ and $\eta^{\prime}=2 \pi$ at $x_{3}=+\infty$. We also apply an external magnetic field of the (11) component of $A$ in the $x_{3}$ direction, with

$$
\int d A^{(11)}=2 \pi
$$

i.e., we put a monopole and an antimonopole at $x_{3}=\mp \infty$.

This configuration provides $B=1$ as one can see from the baryon density in Eq. (C2). One can also understand this as the Witten effect. For the (11) component of $A$, there is an effective $\theta$ term from Eq. (C1),

$$
S_{\theta}=-\frac{N}{8 \pi^{2}} \int A^{(11)} d A^{(11)} d \eta^{\prime},
$$

which varies as a function of $x_{3}$. Therefore, as we move the monopole from $x_{3}=-\infty$ to $x_{3}=+\infty$, the monopole obtains an electric charge $N$ to couple to $A^{(11)}$ by the Witten effect. Since $A^{(11)}=B / N+\cdots$, the dyon carries the baryon number $B=1$. Although the magnetic field is eliminated by this move, the baryon number remains.

Now we consider the situation that the change of the value of $\eta^{\prime}$ happens in a finite region on the $\left(x_{1}, x_{2}\right)$ plane and at a localized location in the $x_{3}$ coordinate. This sheetlike configuration was called the Hall droplet in Ref. [28]. If a monopole goes through the Hall droplet, it becomes a dyon with $B=1$ by the Witten effect. This means that if we put magnets on both sides of the droplet, the magnetic lines cannot just go through the droplet. In order to let the monopole line go through, one needs to throw in a baryon. Conversely, starting from a configuration where the magnetic lines penetrate the droplet, when the magnets are turned off or taken away the system should relax to a state with a finite baryon number. It was discussed in Ref. [28] that the excitation of the edge mode of the droplet corresponds to the baryon state with spin $N / 2$. This state is a good candidate for the remnant of the system.

The flavor quantum numbers of systems can be read off in the same way as for the baryon number. Let us take the cases with $N=3$ and $N_{f}=2$ as in real QCD, where $A^{(11)}$ couples to the current of the up quark. The configuration of the unit magnetic line of $A^{(11)}$ going through the droplet now has the quantum number of the operator $u u u$, i.e., it has the electric charge $Q=2$. This is indeed the same as the baryon discussed in Ref. [28].

Let us consider another example where only one of the diagonal components of the Nambu-Goldstone field has nontrivial configurations:

$$
\begin{aligned}
& \left.\left(\pi^{a} T^{a}+\eta^{\prime}\right)\right|_{x_{3}=-\infty}=\left(\begin{array}{cccc}
0 & & & \\
& 0 & & \\
& & \ddots & \\
& & & 0
\end{array}\right), \\
& \left.\left(\pi^{a} T^{a}+\eta^{\prime}\right)\right|_{x_{3}=+\infty}=\left(\begin{array}{cccc}
2 \pi & & & \\
& 0 & & \\
& & \ddots & \\
& & & 0
\end{array}\right) .
\end{aligned}
$$

When the $U(1)$ baryon (or QED as in the real world) is gauged, the minimal magnetic charge is

$$
\int \frac{d B}{N}=\int d A^{(11)}=\cdots=\int d A^{\left(N_{f} N_{f}\right)}=\frac{2 \pi}{N},
$$

as in the well-known magnetic monopole in grand unified theories $[111,112]$. The Dirac quantization conditions for quarks are satisfied by taking into account the $\mathbb{Z}_{N}$ magnetic flux of $S U(N)$ carried by the monopole $[113,114]$. The configuration that the magnetic line of this monopole (i.e., the 't Hooft line) penetrates the Hall droplet now has the baryon number $B=1 / N$. Therefore, once the magnets are removed, the system should relax to a quark! Again, by the Witten effect the 't Hooft line accompanies a Wilson line when it goes across the droplet. When we turn off the magnetic part, the Wilson line that ends on the sheet remains. There should be a quark at the end point of the Wilson line. Indeed, there is an anyon excitation of the Hall droplet with baryon number $B=1 / N$. (See Ref. [115] for a review.) It is interesting that the quark is described as a soliton made of hadrons!

The discussion here is closely related to the chiral soliton lattice studied in Ref. [116], where the pions develop winding numbers under strong magnetic fields and a chemical potential of baryons. Microscopically, one baryon can be converted into a configuration of a Hall droplet with one unit of magnetic flux penetrating it. 
[1] J. Wess and B. Zumino, Consequences of anomalous Ward identities, Phys. Lett. 37B, 95 (1971).

[2] E. Witten, Global aspects of current algebra, Nucl. Phys. B223, 422 (1983).

[3] G. 't Hooft, Naturalness, chiral symmetry, and spontaneous chiral symmetry breaking, NATO Sci. Ser. B 59, 135 (1980).

[4] Y. Frishman, A. Schwimmer, T. Banks, and S. Yankielowicz, The axial anomaly and the bound state spectrum in confining theories, Nucl. Phys. B177, 157 (1981).

[5] S. R. Coleman and B. Grossman, 't Hooft's consistency condition as a consequence of analyticity and unitarity, Nucl. Phys. B203, 205 (1982).

[6] T. Banks, R. Myerson, and J. B. Kogut, Phase transitions in Abelian lattice gauge theories, Nucl. Phys. B129, 493 (1977).

[7] M. B. Einhorn and R. Savit, Phase transitions in the Abelian Higgs model, Phys. Rev. D 19, 1198 (1979).

[8] M.E. Peskin, Mandelstam 't Hooft duality in Abelian lattice models, Ann. Phys. (N.Y.) 113, 122 (1978).

[9] D. Horn, M. Weinstein, and S. Yankielowicz, Hamiltonian approach to $Z(N)$ lattice gauge theories, Phys. Rev. D 19, 3715 (1979).

[10] A. Ukawa, P. Windey, and A. H. Guth, Dual variables for lattice gauge theories and the phase structure of $Z(N)$ systems, Phys. Rev. D 21, 1013 (1980).

[11] C. Dasgupta and B. I. Halperin, Phase Transition in a Lattice Model of Superconductivity, Phys. Rev. Lett. 47, 1556 (1981).

[12] T. Appelquist and R. D. Pisarski, High-temperature YangMills theories and three-dimensional quantum chromodynamics, Phys. Rev. D 23, 2305 (1981).

[13] M. Gopfert and G. Mack, Proof of confinement of static quarks in three-dimensional U(1) lattice gauge theory for all values of the coupling constant, Commun. Math. Phys. 82, 545 (1981).

[14] T. W. Appelquist, M. J. Bowick, D. Karabali, and L. C. R. Wijewardhana, Spontaneous chiral symmetry breaking in three-dimensional QED, Phys. Rev. D 33, 3704 (1986).

[15] T. Appelquist, D. Nash, and L. C. R. Wijewardhana, Critical Behavior in $(2+1)$-Dimensional QED, Phys. Rev. Lett. 60, 2575 (1988).

[16] T. Appelquist and D. Nash, Critical Behavior in $(2+1)$ Dimensional QCD, Phys. Rev. Lett. 64, 721 (1990).

[17] C. Closset, T. T. Dumitrescu, G. Festuccia, Z. Komargodski, and N. Seiberg, Comments on Chern-Simons contact terms in three dimensions, J. High Energy Phys. 09 (2012) 091.

[18] O. Aharony, Baryons, monopoles and dualities in ChernSimons-matter theories, J. High Energy Phys. 02 (2016) 093.

[19] A. Karch, B. Robinson, and D. Tong, More Abelian dualities in $2+1$ dimensions, J. High Energy Phys. 01 (2017) 017.

[20] A. Karch and D. Tong, Particle-Vortex Duality from 3d Bosonization, Phys. Rev. X 6, 031043 (2016).

[21] N. Seiberg, T. Senthil, C. Wang, and E. Witten, A duality web in $2+1$ dimensions and condensed matter physics, Ann. Phys. (Amsterdam) 374, 395 (2016).
[22] J. Gomis, Z. Komargodski, and N. Seiberg, Phases of adjoint $\mathrm{QCD}_{3}$ and dualities, SciPost Phys. 5, 007 (2018).

[23] D. S. Freed, Z. Komargodski, and N. Seiberg, The sum over topological sectors and $\theta$ in the $2+1$-dimensional $\mathbb{C P}^{1} \sigma$-model, Commun. Math. Phys. 362, 167 (2018).

[24] Z. Komargodski and N. Seiberg, A symmetry breaking scenario for $\mathrm{QCD}_{3}$, J. High Energy Phys. 01 (2018) 109.

[25] D. Gaiotto, Z. Komargodski, and N. Seiberg, Time-reversal breaking in $\mathrm{QCD}_{4}$, walls, and dualities in $2+1$ dimensions, J. High Energy Phys. 01 (2018) 110.

[26] F. Benini, P.-S. Hsin, and N. Seiberg, Comments on global symmetries, anomalies, and duality in $(2+1) \mathrm{d}$, J. High Energy Phys. 04 (2017) 135.

[27] C. Choi, D. Delmastro, J. Gomis, and Z. Komargodski, Dynamics of $\mathrm{QCD}_{3}$ with rank-two quarks and duality, J. High Energy Phys. 03 (2020) 078.

[28] Z. Komargodski, Baryons as quantum hall droplets, arXiv:1812.09253.

[29] V. Bashmakov, J. Gomis, Z. Komargodski, and A. Sharon, Phases of $\mathcal{N}=1$ theories in $2+1$ dimensions, J. High Energy Phys. 07 (2018) 123.

[30] A. Armoni, T. T. Dumitrescu, G. Festuccia, and Z. Komargodski, Metastable Vacua in Large- $N \mathrm{QCD}_{3}$, J. High Energy Phys. 01 (2020) 004.

[31] R. Argurio, M. Bertolini, F. Bigazzi, A. L. Cotrone, and P. Niro, QCD domain walls, Chern-Simons theories and holography, J. High Energy Phys. 09 (2018) 090.

[32] G. W. Moore and N. Seiberg, Taming the conformal zoo, Phys. Lett. B 220, 422 (1989).

[33] S. G. Naculich, H. Riggs, and H. Schnitzer, Group level duality in WZW models and Chern-Simons theory, Phys. Lett. B 246, 417 (1990).

[34] E. Mlawer, S. G. Naculich, H. Riggs, and H. Schnitzer, Group level duality of WZW fusion coefficients and Chern-Simons link observables, Nucl. Phys. B352, 863 (1991).

[35] S. G. Naculich and H. J. Schnitzer, Level-rank duality of the U(N) WZW model, Chern-Simons theory, and 2-D qYM theory, J. High Energy Phys. 06 (2007) 023.

[36] P.-S. Hsin and N. Seiberg, Level/rank duality and ChernSimons-matter theories, J. High Energy Phys. 09 (2016) 095.

[37] M. Unsal, Abelian Duality, Confinement, and Chiral Symmetry Breaking in QCD(adj), Phys. Rev. Lett. 100, 032005 (2008).

[38] M. Unsal, Magnetic bion condensation: A New mechanism of confinement and mass gap in four dimensions, Phys. Rev. D 80, 065001 (2009).

[39] M. Unsal and L. G. Yaffe, Center-stabilized Yang-Mills theory: Confinement and large $\mathrm{N}$ volume independence, Phys. Rev. D 78, 065035 (2008).

[40] M. Shifman and M. Unsal, On Yang-Mills theories with chiral matter at strong coupling, Phys. Rev. D 79, 105010 (2009).

[41] M. Shifman and M. Unsal, QCD-like theories on $R_{3} \times S_{1}$ : A smooth journey from small to large $r\left(S_{1}\right)$ with doubletrace deformations, Phys. Rev. D 78, 065004 (2008).

[42] M. Shifman and M. Unsal, Multiflavor QCD* on $R_{3} \times S_{1}$ : Studying transition from Abelian to Non-Abelian confinement, Phys. Lett. B 681, 491 (2009). 
[43] G. Cossu and M. D'Elia, Finite size phase transitions in QCD with adjoint fermions, J. High Energy Phys. 07 (2009) 048.

[44] J. C. Myers and M. C. Ogilvie, Phase diagrams of SU(N) gauge theories with fermions in various representations, J. High Energy Phys. 07 (2009) 095.

[45] M. Unsal and L. G. Yaffe, Large-N volume independence in conformal and confining gauge theories, J. High Energy Phys. 08 (2010) 030.

[46] D. Simic and M. Unsal, Deconfinement in Yang-Mills theory through toroidal compactification with deformation, Phys. Rev. D 85, 105027 (2012).

[47] E. Thomas and A. R. Zhitnitsky, Topological susceptibility and contact term in QCD. A toy model, Phys. Rev. D 85, 044039 (2012).

[48] M. M. Anber, E. Poppitz, and M. Unsal, 2d affine XY-spin model/4d gauge theory duality and deconfinement, J. High Energy Phys. 04 (2012) 040.

[49] E. Poppitz, T. Schafer, and M. Unsal, Universal mechanism of (semi-classical) deconfinement and thetadependence for all simple groups, J. High Energy Phys. 03 (2013) 087.

[50] M. Unsal, Theta dependence, sign problems and topological interference, Phys. Rev. D 86, 105012 (2012).

[51] P. C. Argyres and M. Unsal, The semi-classical expansion and resurgence in gauge theories: New perturbative, instanton, bion, and renormalon effects, J. High Energy Phys. 08 (2012) 063.

[52] M. M. Anber, S. Collier, E. Poppitz, S. Strimas-Mackey, and B. Teeple, Deconfinement in $\mathcal{N}=1$ super Yang-Mills theory on $\mathbb{R}^{3} \times \mathbb{S}^{1}$ via dual-Coulomb gas and "affine" $\mathrm{XY}$ model, J. High Energy Phys. 11 (2013) 142.

[53] G. Cossu, H. Hatanaka, Y. Hosotani, and J.-I. Noaki, Polyakov loops and the Hosotani mechanism on the lattice, Phys. Rev. D 89, 094509 (2014).

[54] A. Bhoonah, E. Thomas, and A. R. Zhitnitsky, Metastable vacuum decay and $\theta$ dependence in gauge theory. Deformed QCD as a toy model, Nucl. Phys. B890, 30 (2014).

[55] M. M. Anber, E. Poppitz, and B. Teeple, Deconfinement and continuity between thermal and (super) Yang-Mills theory for all gauge groups, J. High Energy Phys. 09 (2014) 040.

[56] G. Bergner and S. Piemonte, Compactified $\mathcal{N}=1$ supersymmetric Yang-Mills theory on the lattice: Continuity and the disappearance of the deconfinement transition, J. High Energy Phys. 12 (2014) 133.

[57] T. Misumi and T. Kanazawa, Adjoint QCD on $\mathbb{R}^{3} \times S^{1}$ with twisted fermionic boundary conditions, J. High Energy Phys. 06 (2014) 181.

[58] X. Li and M. B. Voloshin, Metastable vacuum decay in center-stabilized Yang-Mills theory at large N, Phys. Rev. D 90, 105028 (2014).

[59] T. Iritani, E. Itou, and T. Misumi, Lattice study on QCDlike theory with exact center symmetry, J. High Energy Phys. 11 (2015) 159.

[60] M. M. Anber, E. Poppitz, and T. Sulejmanpasic, Strings from domain walls in supersymmetric Yang-Mills theory and adjoint QCD, Phys. Rev. D 92, 021701 (2015).
[61] M. M. Anber and E. Poppitz, On the global structure of deformed Yang-Mills theory and $\mathrm{QCD}(\operatorname{adj})$ on $\mathbb{R}^{3} \times \mathbb{S}^{1}$, J. High Energy Phys. 10 (2015) 051.

[62] A. Cherman, T. Schafer, and M. Unsal, Chiral Lagrangian from Duality and Monopole Operators in Compactified QCD, Phys. Rev. Lett. 117, 081601 (2016).

[63] M. M. Anber and A. R. Zhitnitsky, Oblique Confinement at $\theta \neq 0$ in weakly coupled gauge theories with deformations, Phys. Rev. D 96, 074022 (2017).

[64] A. Cherman and M. Unsal, Critical behavior of gauge theories and Coulomb gases in three and four dimensions, arXiv:1711.10567.

[65] Y. Tanizaki, Y. Kikuchi, T. Misumi, and N. Sakai, Anomaly matching for the phase diagram of massless $\mathbb{Z}_{N}$-QCD, Phys. Rev. D 97, 054012 (2018).

[66] Y. Tanizaki, T. Misumi, and N. Sakai, Circle compactification and 't Hooft anomaly, J. High Energy Phys. 12 (2017) 056.

[67] K. Aitken, A. Cherman, E. Poppitz, and L. G. Yaffe, QCD on a small circle, Phys. Rev. D 96, 096022 (2017).

[68] T. A. Ivanova, O. Lechtenfeld, and A. D. Popov, NonAbelian sigma models from Yang-Mills theory compactified on a circle, Phys. Lett. B 781, 322 (2018).

[69] M. Hongo, T. Misumi, and Y. Tanizaki, Phase structure of the twisted $S U(3) / U(1)^{2}$ flag sigma model on $\mathbb{R} \times S^{1}$, J. High Energy Phys. 02 (2019) 070.

[70] M. M. Anber and E. Poppitz, Domain walls in high-T SU(N) super Yang-Mills theory and QCD(adj), J. High Energy Phys. 05 (2019) 151.

[71] K. Aitken, A. Karch, and B. Robinson, Deconstructing S-duality, SciPost Phys. 4, 032 (2018).

[72] C. Córdova, D. S. Freed, H. T. Lam, and N. Seiberg, Anomalies in the space of coupling constants and their dynamical applications I, SciPost Phys. 8, 001 (2020).

[73] C. Córdova, D. S. Freed, H. T. Lam, and N. Seiberg, Anomalies in the space of coupling constants and their dynamical applications II, SciPost Phys. 8, 002 (2020).

[74] J. J. Sakurai, Theory of strong interactions, Ann. Phys. (N.Y.) 11, 1 (1960).

[75] M. Bando, T. Kugo, S. Uehara, K. Yamawaki, and T. Yanagida, Is rho Meson a Dynamical Gauge Boson of Hidden Local Symmetry?, Phys. Rev. Lett. 54, 1215 (1985).

[76] M. Bando, T. Kugo, and K. Yamawaki, On the vector mesons as dynamical gauge bosons of hidden local symmetries, Nucl. Phys. B259, 493 (1985).

[77] M. Bando, T. Kugo, and K. Yamawaki, Nonlinear realization and hidden local symmetries, Phys. Rep. 164, 217 (1988).

[78] D. T. Son and M. A. Stephanov, QCD and dimensional deconstruction, Phys. Rev. D 69, 065020 (2004).

[79] T. Sakai and S. Sugimoto, Low energy hadron physics in holographic QCD, Prog. Theor. Phys. 113, 843 (2005).

[80] J. Erlich, E. Katz, D. T. Son, and M. A. Stephanov, QCD and a Holographic Model of Hadrons, Phys. Rev. Lett. 95, 261602 (2005).

[81] L. Da Rold and A. Pomarol, Chiral symmetry breaking from five dimensional spaces, Nucl. Phys. B721, 79 (2005). 
[82] Z. Komargodski, Vector mesons and an interpretation of Seiberg duality, J. High Energy Phys. 02 (2011) 019.

[83] R. Kitano, Hidden local symmetry and color confinement, J. High Energy Phys. 11 (2011) 124.

[84] S. Abel and J. Barnard, Seiberg duality versus hidden local symmetry, J. High Energy Phys. 05 (2012) 044.

[85] A. Armoni and V. Niarchos, Phases of $\mathrm{QCD}_{3}$ from nonSUSY Seiberg duality and brane dynamics, Phys. Rev. D 97, 106001 (2018).

[86] M. Akhond, A. Armoni, and S. Speziali, Phases of $\mathbf{U}\left(\mathbf{N}_{\mathbf{c}}\right)$ $\mathrm{QCD}_{3}$ from type 0 strings and Seiberg duality, J. High Energy Phys. 09 (2019) 111.

[87] C. Vafa and E. Witten, Eigenvalue inequalities for fermions in gauge theories, Commun. Math. Phys. 95, 257 (1984).

[88] A. Sharon, $\mathrm{QCD}_{3}$ dualities and the F-theorem, J. High Energy Phys. 08 (2018) 078.

[89] C. Vafa and E. Witten, Restrictions on symmetry breaking in vector-like gauge theories, Nucl. Phys. B234, 173 (1984).

[90] C. Vafa and E. Witten, Parity Conservation in QCD, Phys. Rev. Lett. 53, 535 (1984).

[91] H. Kouno, T. Misumi, K. Kashiwa, T. Makiyama, T. Sasaki, and M. Yahiro, Differences and similarities between fundamental and adjoint matters in $\mathrm{SU}(\mathrm{N})$ gauge theories, Phys. Rev. D 88, 016002 (2013).

[92] Y. Hosotani, Dynamical mass generation by compact extra dimensions, Phys. Lett. 126B, 309 (1983).

[93] A. Roberge and N. Weiss, Gauge theories with imaginary chemical potential and the phases of QCD, Nucl. Phys. B275, 734 (1986).

[94] S. K. Paul and A. Khare, Charged vortices in Abelian Higgs model with Chern-Simons term, Phys. Lett. B 174, 420 (1986); Erratum, Phys. Lett. B 177, 453(E) (1986).

[95] F. Cuteri, C. Czaban, O. Philipsen, and A. Sciarra, Updates on the Columbia plot and its extended/alternative versions, EPJ Web Conf. 175, 07032 (2018).

[96] O. Domenech, G. Panico, and A. Wulzer, Massive pions, anomalies and baryons in holographic QCD, Nucl. Phys. A853, 97 (2011).

[97] S. Borsanyi et al., Calculation of the axion mass based on high-temperature lattice quantum chromodynamics, Nature (London) 539, 69 (2016).

[98] A. Tomiya, G. Cossu, S. Aoki, H. Fukaya, S. Hashimoto, T. Kaneko, and J. Noaki, Evidence of effective axial U(1) symmetry restoration at high temperature QCD, Phys. Rev. D 96, 034509 (2017); Addendum, Phys. Rev. D 96, 079902(A) (2017).

[99] A. A. Abrikosov, On the Magnetic properties of superconductors of the second group, Zh. Eksp. Teor. Fiz. 32,
1442 (1957) [Sov. Phys. JETP 5, 1174 (1957)], http://www .jetp.ac.ru/cgi-bin/e/index/e/5/6/p1174?a=list.

[100] H. B. Nielsen and P. Olesen, Vortex line models for dual strings, Nucl. Phys. B61, 45 (1973).

[101] E. Witten, Dyons of charge $e \theta / 2 \pi$, Phys. Lett. B 86, 283 (1979).

[102] A. M. Polyakov, Compact gauge fields and the infrared catastrophe, Phys. Lett. B 59B, 82 (1975).

[103] A. M. Polyakov, Quark confinement and topology of gauge groups, Nucl. Phys. B120, 429 (1977).

[104] I. Affleck, J. A. Harvey, L. Palla, and G. W. Semenoff, The Chern-Simons term versus the monopole, Nucl. Phys. B328, 575 (1989).

[105] K.-I. Kondo, M. Tanabashi, and K. Yamawaki, Renormalization in the gauged Nambu-Jona-Lasinio model, Prog. Theor. Phys. 89, 1249 (1993).

[106] Y. Nakahara, M. Asakawa, and T. Hatsuda, Hadronic spectral functions in lattice QCD, Phys. Rev. D 60, 091503 (1999).

[107] P. de Forcrand, M. Garcia Perez, T. Hashimoto, S. Hioki, H. Matsufuru, O. Miyamura, A. Nakamura, I. Stamatescu, T. Takaishi, and T. Umeda (QCD-TARO Collaboration), Meson correlators in finite temperature lattice QCD, Phys. Rev. D 63, 054501 (2001).

[108] E. Laermann and P. Schmidt, Meson screening masses at high temperature in quenched QCD with improved Wilson quarks, Eur. Phys. J. C 20, 541 (2001).

[109] M. Cheng et al., Meson screening masses from lattice QCD with two light and the strange quark, Eur. Phys. J. C 71, 1564 (2011).

[110] R. Kitano, M. Nakamura, and N. Yokoi, Making confining strings out of mesons, Phys. Rev. D 86, 014510 (2012).

[111] G. 't Hooft, Magnetic monopoles in unified gauge theories, Nucl. Phys. B79, 276 (1974).

[112] A. M. Polyakov, Particle spectrum in the quantum field theory, JETP Lett. 20, 194 (1974), http://www.jetpletters .ac.ru/ps/1789/article_27297.shtml.

[113] F. Englert and P. Windey, Quantization condition for 't Hooft monopoles in compact simple lie groups, Phys. Rev. D 14, 2728 (1976).

[114] P. Goddard, J. Nuyts, and D. I. Olive, Gauge theories and magnetic charge, Nucl. Phys. B125, 1 (1977).

[115] D. Tong, Lectures on the quantum hall effect, arXiv:1606 .06687 .

[116] T. Brauner and N. Yamamoto, Chiral soliton lattice and charged pion condensation in strong magnetic fields, J. High Energy Phys. 04 (2017) 132. 